\title{
Cluster observations of magnetic field fluctuations in the high-altitude cusp
}

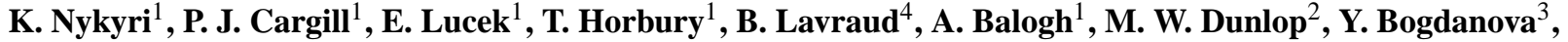 \\ A. Fazakerley ${ }^{3}$, I. Dandouras ${ }^{4}$, and H. Rème ${ }^{4}$ \\ ${ }^{1}$ The Blackett Laboratory, Imperial College, London, UK \\ ${ }^{2}$ Rutherford Appleton Laboratory, UK \\ ${ }^{3}$ Mullard Space science laboratory, University College London, UK \\ ${ }^{4}$ Centre d'Etude Spatiale des Rayonnements, Toulouse, France
}

Received: 11 September 2003 - Revised: 19 February 2004 - Accepted: 3 March 2004 - Published: 14 July 2004

Part of Special Issue "Spatio-temporal analysis and multipoint measurements in space"

\begin{abstract}
High-resolution (22 vector/s) magnetic field data from Cluster FGM instrument are presented for the highaltitude cusp crossing on 17 March 2001. Despite the quiet solar wind conditions, the cusp was filled with magnetic field turbulence for much of the crossing. Large-scale fluctuations show some correlation between spacecraft but the higher frequency fluctuations show no correlation, indicating that the length scales of these waves are smaller than the spacecraft separation $(500 \mathrm{~km})$. In many intervals, there are clear peaks in the wave power around the ion cyclotron frequency $(\sim 1 \mathrm{~Hz})$, and there is some evidence for waves at the first harmonic of this frequency. Both left- and right-hand polarised waves are found, with angles of propagation with respect to the ambient magnetic field that range from parallel to perpendicular. The regions of enhanced magnetic field fluctuations appear to be associated with plasma flows possibly originating from a lobe reconnection site. The most coherent, long lasting wave trains with frequencies close to local ion cyclotron frequency occur at a boundary between a sheared flow and a stagnant plasma.
\end{abstract}

Key words. Magnetospheric physics (magnetopause, cusp and boundary layers; plasma waves and instabilities) - Space plasma physics (nonlinear phenomena; turbulence)

\section{Introduction}

The high-altitude cusps are regions where the magnetosheath plasma has the most direct access to the ionosphere, and their structure is determined by a complex interaction between the shocked solar wind and the geomagnetic field. Prior to the mid-1990s, in situ measurements of the cusp were relatively infrequent, although important results came from the HEOS and Hawkeye missions (e.g. Paschmann et al., 1976; Kessel

Correspondence to: K. Nykyri

(k.nykyri@ic.ac.uk) et al., 1996; Dunlop et al., 2000). The recent Polar and Interball missions have been important in illustrating many examples of cusp physics. Of particular interest has been work on the identification of lobe reconnection (Scudder et al., 2002; Fuselier et al., 2000; Russell et al., 2000), on the extent of the cusp (Zhou et al., 1999, 2000) and magnetic field turbulence (Savin et al., 1998, 2004), which we return to in a moment.

The four spacecraft multi-instrument Cluster mission represents the first of a new generation of magnetospheric physics missions, and is ideal for studying the high- and midaltitude cusps. With four spacecraft one is able to distinguish between temporal and spatial changes and thus build an appropriate three-dimensional picture of the cusp. The range of spacecraft separations between 100 and $10000 \mathrm{~km}$ is also invaluable. There have been a number of studies of cusp encounters which have begun to give a comprehensive picture. Under conditions of northward IMF, Lavraud et al. (2002, 2004) identified a stagnant exterior cusp: an extensive volume of both very low field and flow in the distant cusp region. They pointed out a probable relation with lobe reconnection. Vontrat-Reberac et al. (2003) has studied a cusp encounter on 17 March 2001 at a time of quiet interplanetary conditions and predominantly northward IMF. They showed that there was a significant population of solar wind plasma well inside the magnetopause, whose origin was probably due to plasma streaming earthward from a lobe reconnection site. Finally, Cargill et al. (2004) examined two crossings for southward IMF with a magnetic cusp showing sharp boundaries and rapid sideways motion, but with a superposed diffuse plasma cusp.

This paper addresses magnetic field turbulence in the cusp at low $(<10 \mathrm{~Hz})$ frequencies. There have been measurements of such turbulence since the 1970s (e.g. Scarf et al., 1972), but the Polar mission has provided the most complete data of cusp turbulence prior to Cluster. Savin et al. (1998) did the first two-point study of the cusp/magnetosheath interface, using both Interball-1 and Polar magnetic field measurements. Their results indicate that for northward IMF the turbulence 


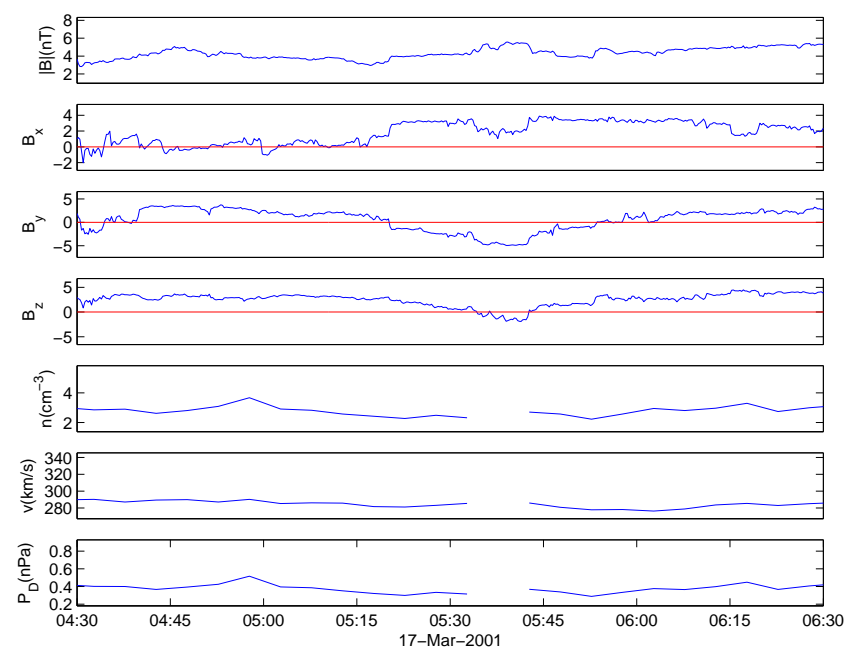

Fig. 1. Lagged (83 min) solar wind data from ACE spacecraft between 04:30-06:30 UT at Cluster on 17 March 2001. The panels from top to bottom show solar wind magnitude, $B_{x}, B_{y}, B_{z}$, SW proton number density, solar wind speed and solar wind dynamical pressure.

is seen inside the main magnetopause current sheets. Chen and Fritz (1998) used Polar magnetometer data to demonstrate well-developed spectra of intense $(\delta B / B$ large) magnetic field turbulence. They associated these fluctuations with the production of energetic particles, but Trattner et al. (1999) presented an alternative interpretation in which the energetic ions originated at the bow shock. Le et al. (2001) examined electromagnetic ion cyclotron waves in the highaltitude cusp using high-resolution (8.33 vectors/s) magnetic field data, and analysed 212 cusp passages at altitudes between 4.8 and $8.8 R_{E}$. They found evidence of narrow-band waves in the frequency range 0.2 to $4 \mathrm{~Hz}$ during 197 cusp encounters, and that the properties of the waves were highly variable, exhibiting both left- and right-handed polarisation in the spacecraft frame.

With a formation of four spacecraft, one can examine turbulence in the cusp in many new ways. For example, correlations between different spacecraft can provide information about the structure of the waves, as well as their origin and convection. An early example of this is due to Rezeau et al. (1993), who studied correlations of the ULF magnetic field fluctuations at the magnetopause measured by two ISEE spacecraft $350 \mathrm{~km}$ apart. Based on the correlation time between the two signals they estimated the convection speed of $55 \mathrm{~km} / \mathrm{s}$ for the magnetic structure. In this paper we will study magnetic field fluctuations during the cusp crossing on 17 March 2001, at a time when the spacecraft separations were approximately $600 \mathrm{~km}$. Nykyri et al. (2003) showed an example of waves during 17 March and in addition, analyzed two other crossings from 2002 when spacecraft separation was $\sim 100 \mathrm{~km}$. Here we will conduct a more detailed analysis of the waves throughout the entire 17 March 2001 cusp crossing.
As noted above, the basic phenomenology of this crossing is now quite well established, enabling us to understand the turbulence in the context of the background plasma and field properties. Section 2 presents a brief outline of the cusp crossing, and the solar wind conditions at that time. Section 3 describes the magnetic field observations in detail, and Sect. 4 outlines the conclusions.

\section{Instrumentation, solar wind conditions and overview of the event}

In this paper we focus on magnetic field fluctuations during the outward cusp crossing of 17 March 2001. The cusp crossing occurs between 05:00 and 07:00 UT, but the interval of 05:00-06:00 UT is of particular interest for magnetic field fluctuations. The overal properties of this crossing have been dicussed by Vontrat-Reberac et al. (2003), so we here provide only a brief summary.

\subsection{Instrumentation}

We use data from three instruments on board Cluster. High resolution (22.4 vectors/second) magnetic field measurements are obtained from the Flux Gate Magnetometer (FGM: Balogh et al., 1997, 2001) from all four spacecraft. Ion plasma measurements are obtained using the Cluster Ion Spectrometry CIS: Rème et al., 2001) experiment. We present data from the Hot Ion Analyses (HIA) on board spacecraft 1 and 3 and the ion COmposition and DIstribution Function analyser (CODIF) on board spacecraft 4 . The plasma velocity moments were available for every $\sim 4 \mathrm{~s}$ (one spin), except from spacecraft 3 , for which the resolution is $\sim 12$ s. From HIA, we show moments for plasma density and velocity. From CODIF, the measurements are shown only for protons. In addition, we show data from the low energy electron analyser (LEEA) from Plasma Electron and Current Experiment (PEACE: Johnstone et al., 1997).

\subsection{Solar wind conditions}

The lagged solar wind conditions observed by the Advanced Composition Explorer (ACE) spacecraft at the L1 (assuming $285 \mathrm{~km} / \mathrm{s}$ constant lag speed) between 04:30-06:30 UT are shown in Fig. 1. During this interval the solar wind had a magnetic field magnitude of approximately $5 \mathrm{nT}, B_{z}$ was mostly pointing northward (except for a brief southward turning at about 05:40 UT in Fig. 1, corresponding to $\sim 04: 17$ UT at ACE) with a magnitude of approximately $3 \mathrm{nT}$. $B_{y}$ was mostly positive with magnitude of $3 \mathrm{nT}$ but turned negative at about 05:17 UT at Cluster for about $35 \mathrm{~min}$. $B_{x}$ was close to $0 \mathrm{nT}$ until $\sim 5: 17 \mathrm{UT}$ at Cluster, after which it turned to positive $\sim 2.5 \mathrm{nT}$.

\subsection{Overview of the event}

Figure 2 shows the spacecraft orbit between 02:00 and 11:00 UT in the GSM X-z plane and for context we show magnetic field lines derived from the Tsyganenko (1989) 


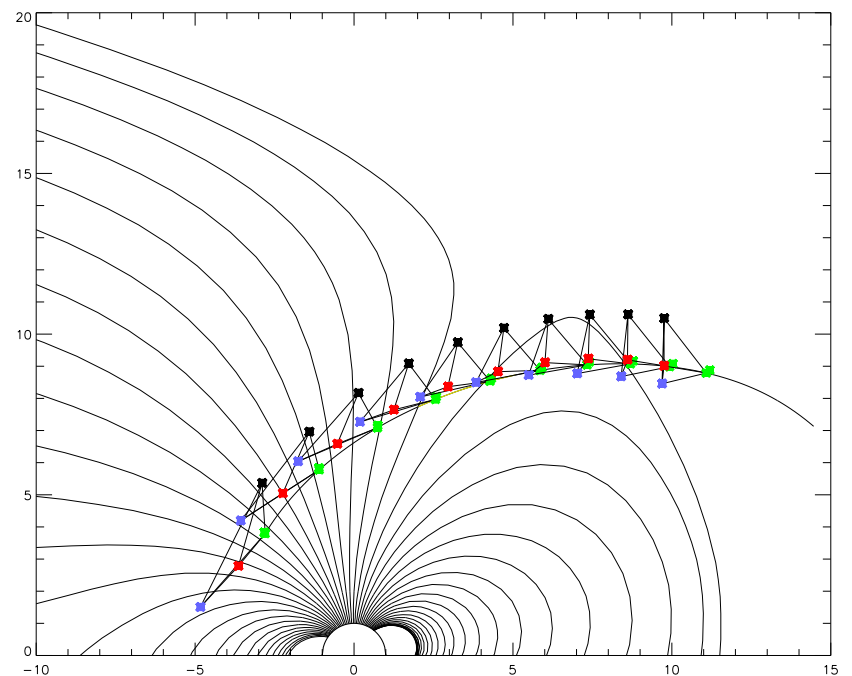

Fig. 2. Cluster trajectory between 02:00-11:00 UT with magnetic field lines from the Tsyganenko (1989) model at 05:00 UT shown for reference. For clarity, the distances between the spacecraft are a factor of 20 larger than in reality.

field model with a $K_{p}$ value of 0 . We use GSM coordinates throughout this event. The Cluster tetrahedron formation is shown at each hour in Fig. 2, with distances magnified by a factor of twenty for clarity. The colour code used here (and throughout this paper) is as follows: black - spacecraft 1 , red - spacecraft 2 , green - spacecraft 3 and blue - spacecraft 4, and for convenience we refer to the four spacecraft as SC1, $\mathrm{SC} 2, \mathrm{SC} 3$ and SC4, hereafter. For reference we note that the formation indicates that any planar discontinuity lying in the y-z plane will be encountered first by SC3, and followed by $\mathrm{SC} 1, \mathrm{SC} 2$ and SC4, respectively. Figure 3 shows the Cluster formation at three times at 05:00 UT, with the three panels showing the projection on the GSM x-y, $x-z$ and $y-z$ planes. The blue arrows originating at each spacecraft are the velocity vectors, and the aqua colored arrows represent the direction of the local magnetic field in the 2-D plots. In the 3-D plot the direction of the background magnetic field is marked with blue arrow. The typical separation between the spacecraft is of the order of $600 \mathrm{~km}$.

Figure 4 shows the electron fluxes parallel to the ambient magnetic field as seen by the PEACE LEEA instrument on all four spacecraft between 05:00-06:12 UT. The cusp is readily identifiable by the onset of low-energy solar wind electrons with energies in the range $\sim 10 \mathrm{eV}$ to $\sim 200 \mathrm{eV}$ just after 05:00 UT. These are seen first by SC3, and then by the other three spacecraft. There are two gaps in electron precipitation at $\sim 5: 32$ and at 5:52 UT. Comparing Fig. 4 to Fig. 2, the cusp entry from Tsyganenko (1989) (at 05:00 UT) is nicely consistent with observations of magnetosheath electrons at around 05:07 UT.
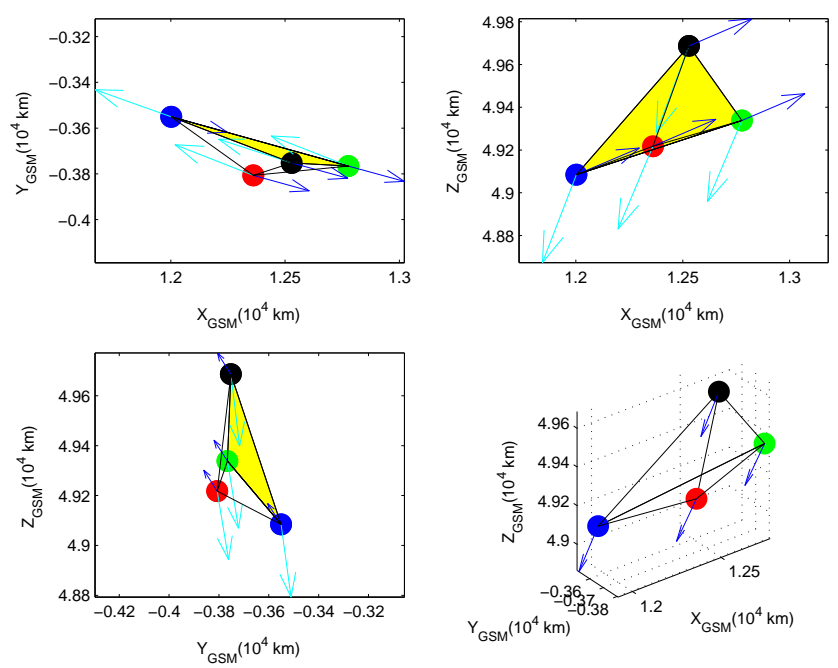

Fig. 3. Cluster tetrahedron formations at $x-y, x-z, y-z-$ planes and in 3-D box (x, y, z) on 17 March 2001 at 05:00 UT. In 2-D plots the aqua arrows indicate the background magnetic field direction and the blue ones the spacecraft velocity vector. In 3 -D plot the aqua colored arrows denote the background magnetic field direction. For clarity, in all plots the distances between the spacecraft are a factor of 20 larger than in reality.

Figure 5 shows a summary of the Cluster ion and spinaveraged magnetic field observations on 17 March 2001 between 05:00 and 06:00 UT. From top to bottom the panels show: plasma density $(\mathrm{N}), \mathrm{x}$-component of the plasma velocity $\left(V_{x}\right)$, the de-trended $\mathrm{x}$-component of the magnetic field $\left(d B_{x}\right), V_{y}, d B_{y}, V_{z}, d B_{z}$, the total velocity $V_{T}$, the detrended total magnetic field $\left(d B_{T}\right)$. The lowest four panels show $B_{x}, B_{y}, B_{z}$ and $B_{T}$. The de-trended magnetic field components are obtained by extracting a linear fit between 05:00 and 06:00 UT from the magnetic field measurements. The transition into the cusp can be seen as an increase in plasma density just after 05:07 UT, consistent with the PEACE spectra. The ion temperature is not shown, but it is fairly constant after cusp entry and varies between 3 and $1.5 \cdot 10^{6} \mathrm{~K}$ between $05: 12$ and 06:00 UT. It is interesting to note that cusp entry is not especially evident in the orientation of the large-scale magnetic field, but can be associated with the onset of enhanced magnetic field fluctuations.

From the viewpoint of this paper, an important feature is the association between plasma flows and magnetic field fluctuations. There are fluctuations in the ion velocity, with maximum tailward velocities of $\sim 125 \mathrm{~km} / \mathrm{s}$, observed at $\sim$ 05:09 by SC1. Between 05:07 and 05:27 UT, the velocity seen by $\mathrm{SC} 1$ and $\mathrm{SC} 4$ undergoes four oscillations with a period of slightly over 4 minutes. The oscillation initially has an amplitude of $200 \mathrm{~km} / \mathrm{s}$, and gradually diminishes such that the plasma becomes stagnant by 05:27 UT. Although there are differences between the velocities seen by all the spacecraft, those from SC3 differ most from the others. For example, at $\sim 05: 19$ UT, SC3 observes almost stagnant plasma while simultaneously $\mathrm{SC} 1$ and SC4 observe velocities of 

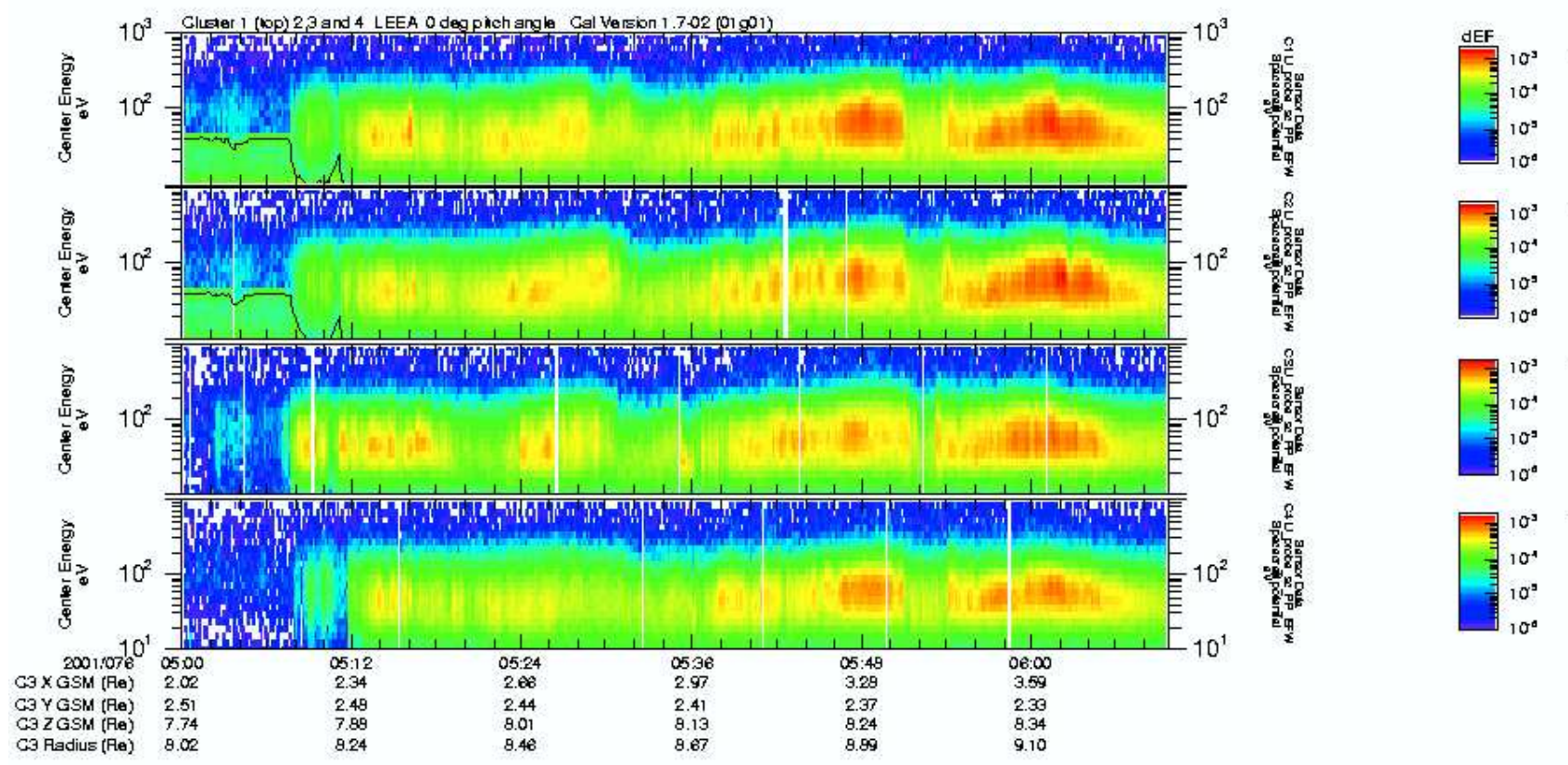

Fig. 4. Field-aligned electron fluxes observed by the LEEA instrument on the PEACE experiment between 05:00-06:00 UT for all spacecraft.

$\sim 100 \mathrm{~km} / \mathrm{s}$. This is indicative of shears in the plasma velocity on scales of the spacecraft separation or less. It is clear from the de-trended data shown in Fig. 5 that the magnetic field oscillations correlate with the enhanced plasma velocities. For example, when the plasma becomes stagnant at $\sim 05: 27$ UT, the magnetic field oscillations disappear. At 05:35 UT the velocity fluctuations reappear, but with smaller amplitudes than during the initial cusp entry, and are accompanied by magnetic field oscillations which show more coherent features than earlier. (We also note that between 05:35 and 05:42 UT, there are large-scale magnetic field oscillations that resemble flux transfer events with bipolar signatures in the component of the magnetic field in the minimum variance direction.) Except for the region of stagnant plasma between 05:4805:50 UT, small amplitude velocity fluctuations are present between 05:35-05:57 UT, after which plasma becomes stagnant again. There is also a persistent level of magnetic field fluctuations associated with these flows.

We can understand the formation of these different regions in terms of the IMF conditions. Prior to 05:30 UT the IMF $B_{z}$ is northward (see Fig. 1), and precipitating solar wind plasma can be seen in the field-aligned electrons, as well as the fluctuations in plasma velocity in Fig. 5. At $\sim 05: 30$ UT the IMF $B_{z}$, and at $\sim 05: 17$ UT $B_{y}$, rotate from positive to negative which can lead to reconnection at sub-solar point and the subsequent dawnward-motion of reconnected flux tubes. Depending on how fast the cusp can adjust to the changing IMF conditions, it could be that during the interval of stagnant plasma, between 05:24 and 05:35 UT, the cusp topology is changing, and direct entry of solar wind plasma is inhibited. This can also been seen in Fig. 4 as a gap of solar wind electron precipitation between 05:30 and 05:36 UT. Af- ter $\sim 05: 40$ UT the IMF $B_{z}$ rotates back to being positive, and $B_{y}$ also rotates slightly later at $\sim 05: 50 \mathrm{UT}$. These changes in the IMF would correlate with the reappearance of precipitating solar wind plasma at 05:38 UT and as enhanced plasma velocity in Fig. 5 at 05:35 UT if we assume a 5-min uncertainity in lag time. If we assume this uncertainity, the second stagnant plasma interval at $\sim 05: 49$ UT doesn't seem to have any solar wind signature.

In summary, from the viewpoint of magnetic field fluctuations, the cusp between 05:00-06:00 UT can be divided in two types of region. Firstly, there are regions with fluctuating plasma velocity, and velocity gradients on the scale of the spacecraft separation or less. These regions have a significant level of magnetic field fluctuations that is probably driven by the precipitating solar wind plasma. Secondly, there is a stagnant cusp (i.e. small or no plasma flows) and an absence of magnetic field oscillations. In the following sections we refer to these regions as the shear-flow and stagnant cusp, respectively.

\section{Observations of cusp magnetic field fluctuations}

\subsection{Overview}

It can be seen in Fig. 5 that large-scale magnetic field fluctuations with amplitudes of $\sim 4-5 \mathrm{nT}$ correlate with regions of precipitating magnetosheath plasma. Our analysis of the high-resolution FGM magnetic field data shows that higher frequency magnetic field fluctuations with frequency $(f)$ close to local ion cyclotron frequency $f_{i}=\Omega_{i} / 2 \pi=e B /\left(m_{i} 2 \pi\right)$ are also present. In this study we will focus on the analysis of magnetic field fluctuations 




Fig. 5. Overview plot of the Cluster plasma and magnetic field observations on 17 March 2001 at 05:00-06:00 UT. Panels are from top to bottom as follows: plasma density $(N)$, x-component of the plasma velocity $\left(V_{x}\right)$, x-component of the de-trended magnetic field $\left(d B_{x}\right), V_{y}$, $d B_{y}, V_{z}, d B_{z}$, total velocity $\left(V_{T}\right)$, de-trended total magnetic field component $\left(d B_{T}\right), B_{x}, B_{y}, B_{z}, B_{\text {tot }}$.

around $f_{i}$, and divide the analysis into the following spatial regions: Sect. 3.2, the strong shear-flow cusp between 05:07 and 05:25 UT; Sect. 3.3, the stagnant cusp between 05:25 and 05:35 UT and Sect. 3.4, the moderate shear-flow cusp between 05:35 and 05:56 UT. Here we present a detailed analysis of the wave properties during 150 wave intervals between 05:00-06:00 UT. Waves at the inner magnetosphere- cusp boundary, the transition to the dayside magnetosphere, and ULF fluctuations seen in the dayside magnetosphere will be discussed elsewhere. 

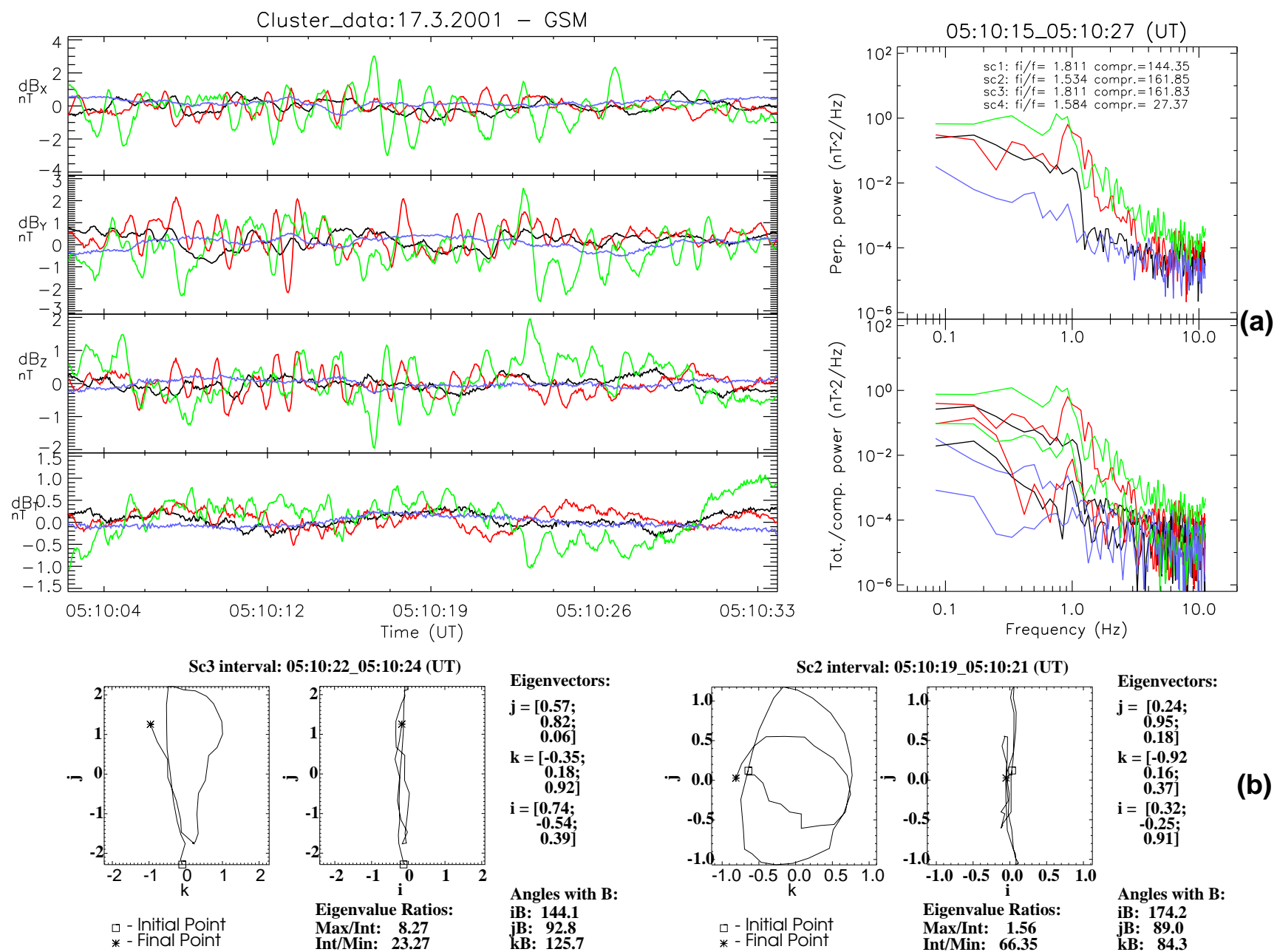

(b)

Fig. 6. (a) De-trended magnetic field components between 05:10:01 and 05:10:34 UT for all Cluster spacecraft (left). On right-hand side the upper plot shows the power in fluctuations perpendicular to the magnetic field at 05:10:15-05:10:27 UT for all 4 spacecraft. The total and parallel power are plotted on bottom, with the total power being the upper curve for each spacecraft. Panel (b) shows magnetic field hodograms for waves between 05:10:22 and 05:10:24 UT observed by SC3 (left) and between 05:10:19 and 05:10:21 UT by SC2 (right). Other information shown on the figure includes the values of $R$ and $C$ (in the power spectra), the minimum variance eigenvalue ratios, eigenvectors and wave propagation angles (next to the hodograms).

\subsection{Strong shear flow cusp: 05:07-05:25 UT}

As an example of waves in the strong shear interval, the left panel of Fig. 6a shows de-trended magnetic field components in the interval 05:10:01-05:10:33 UT for all spacecraft. Each window is locally de-trended by extracting a linear fit from the magnetic field measurements. One can see evidence of significant fluctuations at $\mathrm{SC} 2$ and $\mathrm{SC} 3$, but a much quieter magnetic field at $\mathrm{SC} 1$ and $\mathrm{SC} 3$. We have calculated the crosscorrelation coefficients for the de-trended field components for each spacecraft pair, and find very poor cross-correlation coefficients for all lag times.

We now calculate the power in each de-trended field component using a 12-s window (the Nyquist frequency is $11.2 \mathrm{~Hz}$ ). The window size is determined by our interest in ion cyclotron waves. The ion cyclotron frequency varies from $\sim 1.6 \mathrm{~Hz}$ to $\sim 0.75 \mathrm{~Hz}$ between 05:00 UT and 06:00 UT giving optimal window lengths of between 5 and $12 \mathrm{~s}$ at 05:00, and between 11 and $25 \mathrm{~s}$ at 06:00 UT. Window sizes of $7,8,10$ and $15 \mathrm{~s}$ were also used to test the consistency of the results. We note that the length of the time series (12s) can give errors to the lowest spectral densities (below $0.3 \mathrm{~Hz}$ or so), but the frequency range under interest here (close to $1 \mathrm{~Hz}$ ) should be unaltered by this. Before evaluating the fast Fourier transform, a Hanning window was applied to the detrended data set, in order to make the signal continuous. The total power is then the trace of the power spectral matrix: $P_{\text {tot }}=P_{x}+P_{y}+P_{z}$. The perpendicular power is then obtained by extracting the power along the mean magnetic field (the parallel power) from the total power: $P_{\text {perp }}=P_{\text {tot }}-P_{\text {parallel }}$.

To examine the compressibility of the waves, the ratio of the amplitudes of the peaks in the perpendicular and parallel power was calculated. Defining this ratio as the quantity $C$ (coefficient compr. in figures), a value of $C>1$ implies 
that the wave is predominately transverse. We also calculate the ratio $R=f_{i} / f$, where $f_{i}$ is the local ion gyrofrequency and $f$ the frequency corresponding to the peak value of the power spectrum. For parallel-propagating Alfvén/ion cyclotron waves in a cold electron-proton plasma, we would always expect $R>1$. There is no restriction on the value of $R$ for the fast/magnetosonic/whistler branch. Values of $R$ and $C$ are shown on the power spectra in each figure.

The right panels of Fig. 6 show the power in fluctuations perpendicular to the magnetic field (top panel), and the total power, and power parallel to the magnetic field, (bottom) between 05:10:15 and 05:10:27. In the lower panel, the top and bottom curves with the same colour correspond to the total and parallel power, respectively. It can be seen that the peak power differs very considerably between SC2 and SC3 and the other two spacecraft, indicative of localised bursts of turbulence, and confirming the impressions of Fig. 6a.

As an example of this localisation, between 05:10:01 and 05:10:34 UT SC2 and SC3 see several incoherent wave packets that are not seen by $\mathrm{SC} 1$ and $\mathrm{SC} 4$. For the interval 05:10:15-05:10:27 UT, we find $R=1.5$ (1.8) for SC2 (SC3), and $C=162$ for both spacecraft, indicating very transverse waves with peak power below $f_{i}$. Of course, Doppler effects can affect the observed frequency in the spacecraft frame since we have $\omega_{\mathrm{obs}^{\prime}}=\omega+\mathbf{k} \cdot \mathbf{V}$, where prime denotes the spacecraft frame. Thus the Doppler shift can make the observed frequency of the left-hand ion cyclotron mode appear smaller (larger) than it would be in the plasma frame, if the plasma flow velocity is anti-parallel (parallel) to the wave vector. If we assume that field and flow are parallel (Fig. 5), then in these cases the frequency of the observed waves has been increased, but in the absence of a determination of the wavelength, we cannot quantify this further.

We now use minimum variance analysis (Sonnerup and Scheible, 1998) to present the wave magnetic field in directions of maximum $(j)$, intermediate $(k)$ and minimum $(i)$ variance. In order to define whether the wave magnetic field is left-hand (ion sense: the Alfvén/ion cyclotron wave) or right-hand (electron sense: the magnetosonic/whistler wave) polarized, we need to know the angle between the background magnetic field and minimum variance direction, defined as $\theta_{k B}$. The lower panels of Fig. 6b show sample wave magnetic field hodograms during this interval for SC3 (left) and $\mathrm{SC} 2$ (right) in $\mathrm{k}-\mathrm{j}$ and $\mathrm{i}-\mathrm{j}$ planes. The initial point on the hodogram is denoted by a square and the end point by an asterisk. In the k-j plots, the minimum variance direction points into the plane and, therefore, the counter clockwise rotation in $\mathrm{k}-\mathrm{j}$ plane corresponds to the clockwise rotation around minimum variance axis. Further information about the minimum variance analysis is shown next to the hodograms (see figure caption for more details).

For SC3 we find $\theta_{k B}=144^{\circ}$, so that the ambient magnetic field comes out of the $\mathrm{k}-\mathrm{j}$ plane. The ratio between maximum and intermediate eigenvalues is 8.27 for SC3, which gives an ellipticity $(\sqrt{\operatorname{Int} / \text { Max }})$ of 0.35 (a circularly (linearly) polarised wave would have a ratio of $1(0)$ ). Thus, for the interval in the hodogram, the wave magnetic field rotates clockwise around the ambient magnetic field in the k-j plane, so that the wave is left-hand polarized in spacecraft frame, and therefore belongs to the Alfvén/ion cyclotron branch. In contrast the hodogram from SC2 shows a right-hand polarized wave with $\theta_{k B}=174^{\circ}$ and an ellipticity of 0.8 , so that the wave belongs to the magnetosonic/whistler branch. (There is a $180^{\circ}$ ambiguity of the minimum variance direction, and so we cannot say whether this wave propagates closely antiparallel or parallel with respect to the background magnetic field. From now on we will use the value of $\theta_{k B}$ as expressed in each hodogram plot to define wave polarisation, but in the text we will refer to $\theta_{k B}$ as the magnitude of the angle between minimum variance and background magnetic field direction.)

These results indicate that the wave power at closelyseparated spacecraft can differ by orders of magnitude, and that when waves are seen, their properties differ significantly on the scale of the spacecraft separation. The configuration shown in Fig. 3 indicates that each of the spacecraft are sampling different bundles of magnetic flux. If one accepts that the waves are generated by field-aligned plasma flows, then clearly each spacecraft is passing through different plasma environments. The differences in the plasma flows discussed earlier could lead to different levels of power, but this conjecture will need the full analysis of plasma distributions for verification.

The largest amplitude waves are seen at SC2 and SC3 which are closest to each other at the y-z-plane. This may indicate that they are generated in a narrow range of GSM y-z space locally, or remotely, and then convected to the spacecraft. However, their local properties are very different. We would expect to see a correlation between SC2 and SC3 if the spacecraft separation vector is aligned with the wave propagation direction, and the wavelength is of the order of the spacecraft separation. The waves at SC2 are almost parallel propagating, but Fig. 3 indicates that the spacecraft separation vector between $\mathrm{SC} 3$ and SC2 is at a very oblique angle with respect to the background magnetic field. Thus, if the waves are generated locally, there is no reason why those at $\mathrm{SC} 2$ should also appear at $\mathrm{SC} 3$, a result confirmed by the lack of cross-correlation.

The opposing polarisations seen at $\mathrm{SC} 2$ and $\mathrm{SC} 3$ are also suggestive of independent generation processes. Here one needs to consider Doppler effects due to motion of the background plasma. Since there are no plasma measurements for spacecraft 2 , the discussion is necessarily incomplete. In the plasma frame, the phase velocity of the wave is $v_{p h}=v_{p h}^{\prime}-V \cos \left(\theta_{k V}\right)$, where $v_{p h}^{\prime}$ is the phase velocity in spacecraft frame, $V$ is the plasma flow velocity and $\theta_{k V}$ the angle between the flow velocity and the wave propagation direction. The Alfvén velocity during this interval for SC3 is $\sim 630 \mathrm{~km} / \mathrm{s}$, almost five times larger than the plasma flow velocity, so that Doppler effects can only have a small effect on the observed wave polarization in this instance, provided $v_{p h} \simeq V_{A}$. For right-handed waves, $v_{p h}$ exceeds $V_{A}$ as one approaches $R=1$, so that the polarisation seen by $\mathrm{SC} 2$ is unlikely to change. For left-handed waves, $v_{p h}$ drops below 

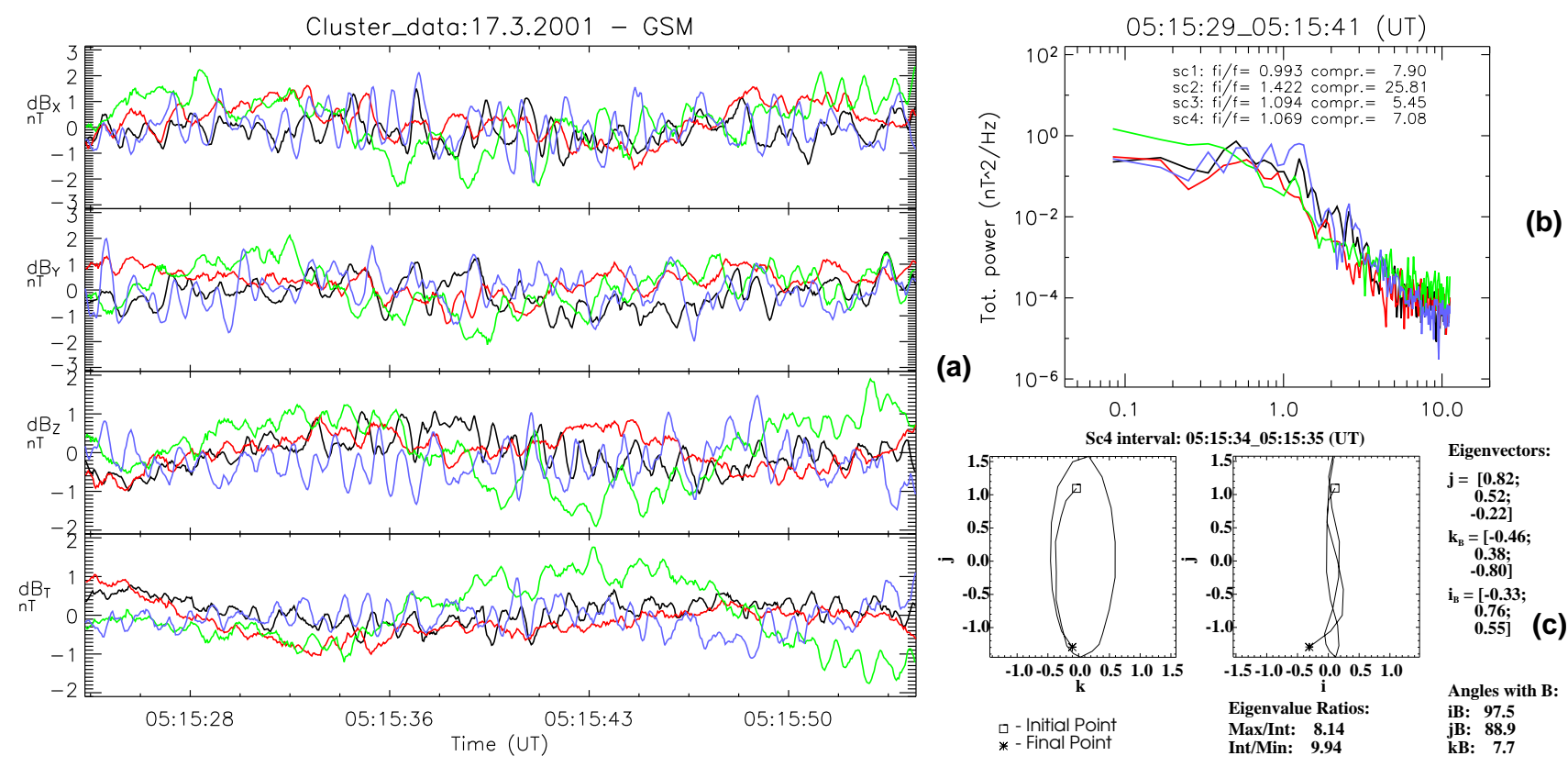

Fig. 7. (a) De-trended magnetic field components between 05:15:20-05:15:58 UT for all Cluster spacecraft (left). The upper right-hand side shows the total power in the fluctuations at 05:15:29-05:15:41 UT for all 4 spacecraft. The magnetic field hodogram between 05:15:3405:15:35 UT observed by SC4 is plotted in panel (c). Other details are as in Fig. 6.

the Alfvén speed as the frequency increases, eventually approaching zero at $f_{i}$, but this is still unlikely to account for a change in polarisation given the small ratio of the plasma velocity to the Alfvén speed.

We analysed all other wave intervals for SC3 and SC2 between 05:10:01-05:10:34 UT and found that the overall wave properties observed by both spacecraft are similar: both spacecraft found left- and right-handed waves with ellipticities varying from 0.28 to 0.64 and magnitudes of propagation angles varying from 6 to $44^{\circ}$ with respect to the background field. There was no clear correlation between ellipticity, polarity and propagation direction.

Figure 7 presents results from the interval 05:15:2505:15:54 UT in the same format as Fig. 6. Figure 7b shows the total spectral power between 05:15:29 and 05:15:41 UT. In this case, the power is similar at all the spacecraft, but SC4 now sees a clear peak around $f_{i}(R=1.07)$. This peak arises from several incoherent bursts of wave activity with a maximum amplitude of $\sim 3 \mathrm{nT}$ that are also evident in the detrended data. The cross-correlation coefficients between SC4 and other three spacecraft are again small. Figure $7 \mathrm{c}$ shows a hodogram from this interval with a very oblique propagation angle $\left(\theta_{k B}=82^{\circ}\right)$, an ellipticity of 0.35 , and $C=7$. Compared to the previous interval, the importance of the transverse wave power is diminished, while the overall power remains similar.

Since the waves observed by SC4 propagate almost perpendicular to the background magnetic field, and the wave frequency is within $6 \%$ of the ion gyrofrequency, we expect the right-hand wave during the interval 05:15:34-05:15:35 to belong to the fast/magnetosonic mode. However, the ob- served transverse ratio of 7 seems large for such a wave, which should be quite compressive. For example, KraussVarban et al. (1994) demonstrated that compressibility approaches one in the case of nearly perpendicular propagation of the magnetosonic mode.

The velocity profiles between 05:15:20-05:15:54 UT indicate that there are significant velocity shears on at least the length scale of the spacecraft separation: SC4 observes a reasonably steady $\mathbf{V}=(-50,50,-130) \mathrm{km} / \mathrm{s}$ in GSM coordinates, whereas $\mathrm{SC} 1$ observes nearly stagnant plasma $\mathbf{V}=(0,-10,0) \mathrm{km} / \mathrm{s}$ until $\sim 05: 15: 27$ UT after which the magnitude of the velocity components gradually increases but without reaching the magnitudes of those observed by SC4. However, SC3 observes stagnant plasma, and only sees an increase in fluctuations after when the plasma velocity slightly increases. This can be seen as further evidence for the role of velocity shear in the generation of these waves.

A final set of results for the strong flow interval is shown in Fig. 8 between 05:23:06 and 05:23:40 UT. Figure 8b shows the total power seen by all spacecraft between 05:23:17 and 05:23:29 UT. There is now a strong peak around $f_{i}$ for $\mathrm{SC} 2$, slightly lower power at SC1 and SC4, and a low level of fluctuations at SC3. SC2 now sees the clearest wave packets with $R=1.3$ and $C \sim 5$, slightly less than during the previous interval. Again, there is no correlation between magnetic field measurements from different spacecraft pairs. Figure $8 \mathrm{c}$ shows hodograms for $\mathrm{SC} 2$ in the interval 05:23:22-05:23:23 UT revealing a right-hand polarized wave with $\theta_{k B}=81^{\circ}$ and an ellipticity of 0.47 . Like the previous interval, we expect this wave to belong to the magnetosonic branch, but again, the transverse ratio $>1$ is not 
consistent with analytical calculations using the kinetic theory of Krauss-Varban et al. (1994). There are again plasma shears with SC 3 observing a flow of $\sim 20 \mathrm{~km} / \mathrm{s}$, and SC1 and $\mathrm{SC} 4$ flows of the order of $60 \mathrm{~km} / \mathrm{s}$.

\subsection{Stagnant cusp: 05:25-05:35 UT}

By 05:25 the plasma becomes nearly stagnant and the larger scale magnetic field fluctuations are no longer present. However, there is an approximately four minute interval from 05:25 to 05:29 UT, where Cluster observes several smallamplitude wave packets with a frequency close to the local ion cyclotron frequency. De-trended magnetic field components between 05:25 and 05:27 UT for all four spacecraft are shown in Fig. 9a and between 05:27 UT and 05:29 in Fig. 9b. Between 05:25 and 05:27 UT there are recognisable small amplitude (below $0.5 \mathrm{nT}$ ) wave packets seen first by SC2 and then by the other 3 spacecraft. Between 05:27 and 05:29 UT SC1 and SC2 observe several very coherent wave packets with maximum amplitudes of $\sim 1 \mathrm{nT}$. There is no good correlation between the time series from any spacecraft pair during each two-minute interval.

The left column of Fig. 10 shows the total power in the magnetic field fluctuations for four 12-s intervals: (a) 05:25:29-05:25:41 UT, (b) 05:26:09-05:26:21 UT, (c) 05:27:54-05:28:06 UT and (d) 05:27:54-05:28:06 UT. The right column shows examples of hodograms obtained during each of these intervals from SC2 for the first two and SC1 for the others. In general, it should be noted that the peaks in the power are considerably lower (by up to an order of magnitude) than in the strong flow interval discussed above: given the almost stagnant plasma, this can be attributed to the lower amount of free energy available to generate the waves. A second point to note is that as before, the power levels at the different spacecraft differ considerably. Finally, we note the apparent presence in many of the spectra of two peaks, one at the ion cyclotron frequency and the other at its first harmonic.

In the first interval the peaks in the spectra seen by SC2 occur at both $R=0.84$ and the second harmonic, $R=0.42$. The waves are strongly transverse $(C=33)$. The amplitude of the first harmonic is $\sim 10$ times smaller than the amplitude of the main peak. In contrast, while $\mathrm{SC} 1$ and $\mathrm{SC} 3$ observe transverse waves with peaks at $R=0.81$ and 0.73 , respectively, there are no clear peaks at the first harmonic. The hodogram shows that $\mathrm{SC} 2$ saw a right-handed wave in this interval with an ellipticity of 0.6 and $\theta_{k B}=43^{\circ}$. SC1 also sees two righthanded waves with ellipticities of 0.56 and 0.61 and $\theta_{k B}$ of 46 and $26^{\circ}$, respectively.

During the second interval all four spacecraft see clear peaks in power around the ion cyclotron frequency, with the highest power observed again by $\mathrm{SC} 2$ at $R=0.89$. SC3 observes a peak at $R=1.15$, and SC1 and SC4 at $R=0.98$ and 0.86 , respectively. SC1 and SC4 also see the first harmonic at $R=0.5$ and 0.42 , respectively, but this is not so clear at $\mathrm{SC} 2$. A sample hodogram comes from $\mathrm{SC} 2$ and shows a lefthanded wave with ellipticity of 0.87 and $\theta_{k B}=16^{\circ}$.
We have also analysed hodograms from eight other intervals between 05:26:09 and 05:26:21 UT. In the interval 05:26:09-05:26:16 UT, SC2 continuously observes left-hand polarized waves. $\theta_{k B}$ varies between 6 and $22^{\circ}$ and the ellipticities are in the range 0.53-0.87. Between 05:26:12 and 05:26:14 UT, SC3 also observes a left-hand polarized wave with $\theta_{k B}=17^{\circ}$ and an ellipticity of 0.65 , similar to SC2. Between 05:26:17 and 05:26:21 UT SC2 observes two righthand polarized waves with ellipticities of 0.9 and 0.85 and with $\theta_{k B}=12$ and $23^{\circ}$. Between 05:26:20 and 05:26:21 UT, SC1 observes a left-handed wave with $\theta_{k B}=37^{\circ}$ and an ellipticity of 0.54 . One thus has a remarkable range of wave properties occurring over very small temporal and spatial intervals. Observations of left-hand polarized waves at SC1 and $\mathrm{SC} 2$ are puzzling, since left-handed waves should not have $R<1$, and in a stagnant plasma, Doppler shifts cannot be invoked. However, Krauss-Varban et al. (1994) demonstrated, using kinetic theory, that in low beta plasma $(\beta=0.2)$ the dispersion curve of the slow/sound mode will cross the disperison curve of the ion cyclotron mode. In low beta plasma the slow/sound mode is left-hand polarized. The plasma beta during this interval is $\sim 0.1$, so our observed left-handed waves above the ion cyclotron frequency could be slow/sound mode waves, if their wave length is $\sim$ smaller than $200 \mathrm{~km}$. Since we don't see very good correlations between the signals seen at different spacecraft whose separation is $\sim 600 \mathrm{~km}$, this could well be possible. However, the existence of slow/sound mode would imply that they are not damped, which might be unrealistic.

In the third interval, SC2 and SC1 both observe strong peaks in the power at $R=0.89$ and 0.91 , respectively, as well as the second harmonic, with an amplitude about 5 times smaller than the dominant peak. SC3 and SC4, on the other hand, see a much lower level of fluctuations. At SC1 and SC2 the waves are strongly transverse (C=30 (SC1) and 128 (SC2)). The hodogram shows a right-hand wave seen by SC1 with ellipticity of 0.7 and $\theta_{k B}=10^{\circ}$. We have also analysed nine intervals between 05:27:19 and 05:27:31 UT for SC2 and SC1, and except for a left-hand wave observed between 05:27:19 and 05:27:21 UT by SC2; all the other wave intervals have right-hand polarised waves. The propagation angles vary between 3 and $37^{\circ}$ and ellipticites between 0.55 and 0.75 . Both spacecraft observe waves during the same intervals with very similar properties, but the correlation coefficients between the time series of SC1 and SC2 are poor. In this case, Fig. 3 shows that SC1 and SC2 are $\sim 490 \mathrm{~km}$ apart and are closest to each other in the $\mathrm{x}$-y-plane but are not located along the same magnetic field line.

In the final interval SC1, SC2 and SC3 all observe strong peaks at $R=0.9$ and the second harmonic. The sample hodogram from SC1 reveals a right-hand, almost circularly polarized wave with ellipticity of 0.94 and $\theta_{k B}=4^{\circ}$. At the same time $\mathrm{SC} 2$ observes a left-hand wave with ellipticity of 0.63 and $\theta_{k B}=23^{\circ}$.

We have also analysed eight intervals between 05:27:54 and 05:28:06 UT. During these intervals the waves are all right-hand polarized, $\theta_{k B}$ varies between 1 and $42^{\circ}$ 

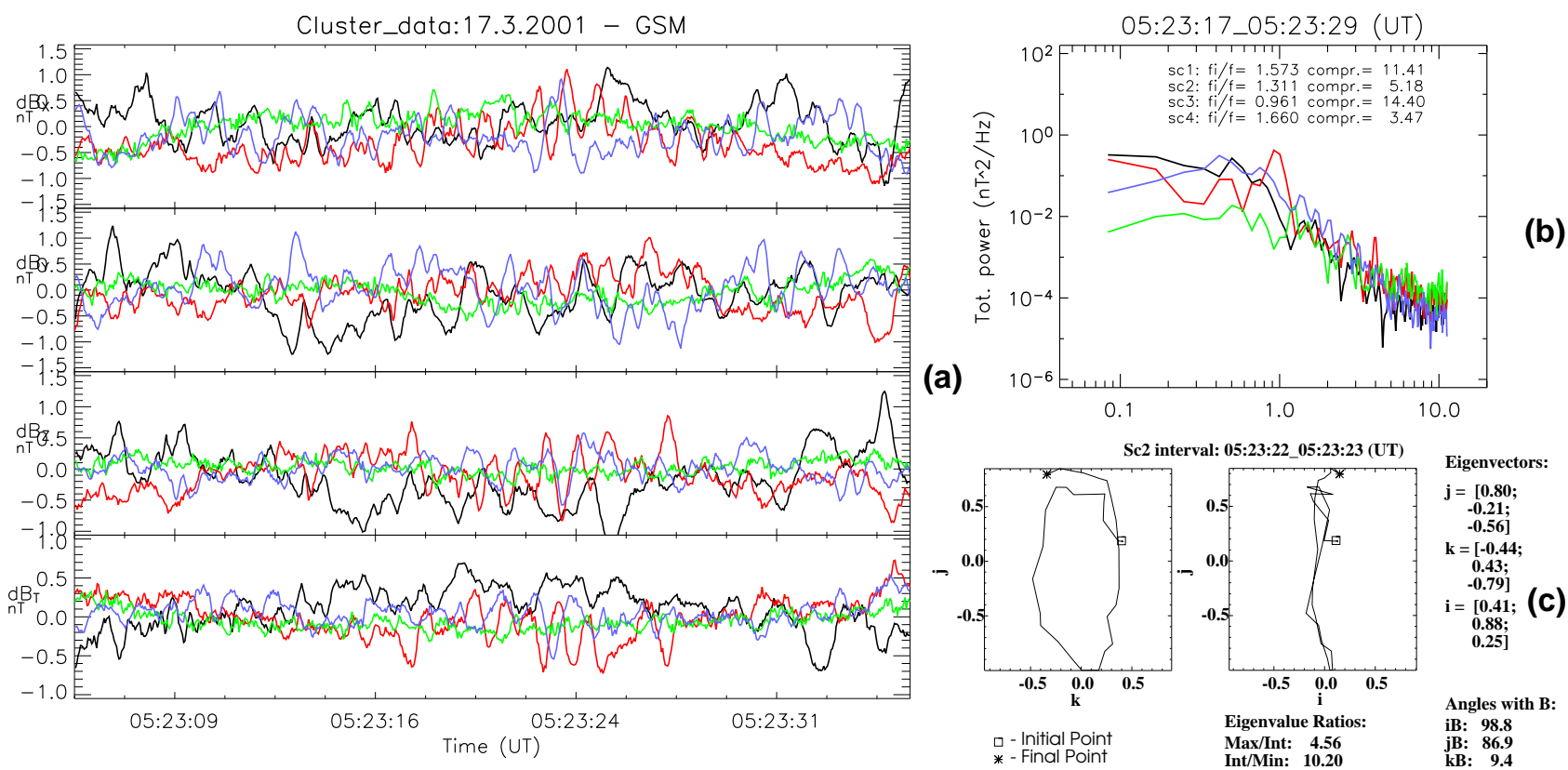

Fig. 8. (a) De-trended magnetic field components between 05:22:50-05:23:42 UT for all Cluster spacecraft (left). On right-hand side are total power in fluctuations (top) to the magnetic field at 05:23:17-05:23:29 UT for all 4 spacecraft. Hodogram of the wave magnetic field between 05:23:22-05:23:23 UT observed by SC2 is plotted on panel (c).

and ellipticities between 0.54-0.94. The cross correlation coefficients between spacecraft pairs during 05:27:5405:28:06 UT are all small, but if we take only a 5-second interval between 05:27:55-05:28:00 UT, we obtain relatively good correlation coefficients (CC) between the spacecraft pairs. However, the small lag times $(<1 \mathrm{~s})$ suggest that these are not a statement of coherence between the spacecraft, but rather are the coincidental local observation by two spacecraft of localised wave packets.

Figure 11 shows wave properties during 75 wave intervals observed by all 4 spacecraft between 05:25 and 05:29 UT. We automated the search for the wave intervals using 1-2-s windows. Only intervals with clear polarization and ellipticity greater than 0.4 were chosen. Each point corresponds to a 1-2-s wave intervals, and we show the magnitude of $\theta_{k B}$ as a function of ellipticity. Squares and triangles represent right-hand and left-hand polarised waves, respectively, and the usual colour coding is used. All the waves have $\theta_{k B}<60^{\circ}$ and most have $\theta_{k B}<40^{\circ}$. There is no clear correlation between ellipticity, propagation direction or polarization of the waves. Between 05:29 and 05:33 UT the plasma is almost completely stagnant. Although there exist some very low amplitude fluctuations, there are no identifiable higher frequency waves during this interval.

\subsection{Moderate shear flow cusp: 05:35-05:54 UT}

Beyond the stagnant cusp region, we have identified the intervals between 05:33 and 05:45 UT and 05:49 and 05:54 UT as the moderate shear flow cusp. Larger scale magnetic field oscillations occur here, with bigger amplitudes than during the stagnant cusp. Similarly to the shear flow cusp, there are intervals where recognisable wave packets are embedded in the larger scale oscillations occurring in the stagnant plasma regions between 05:45 and 05:49 UT.

Figure 12 shows the de-trended magnetic field between 05:45 and 05:47 UT (a) and 05:47 and 05:49 UT (b). The cross-correlation coefficients are again poor for all spacecraft pairs, despite the fact that $\mathrm{SC} 1$ and $\mathrm{SC} 4$ are located approximately $735 \mathrm{~km}$ apart along the same field line. In the first interval, one can see that the fluctuation level differs greatly between the spacecraft, with wave packets appearing and vanishing at each spacecraft seemingly without any relation to the others. In the second interval, one has a more sustained level of fluctuations at all the spacecraft, but again, regions of more intense pulses appear from time to time.

Figure 13 presents power spectra and hodograms from four sample intervals and is in the same format as Fig. 10. We see now that the peak power in the waves is somewhere between the strong shear and stagnant cases, presumably a reflection of the power available to generate the waves. There are clear peaks close to $f_{i}$ (and sometimes its harmonics) in all intervals. As in the other regions presented above, there are major differences in the power seen by the various spacecraft.

In the first interval SC3 observes waves with peaks in power at $R=0.82, C=10$ and a small peak at first harmonic. The hodogram shows a right-hand polarized wave with $\theta_{k B}=52^{\circ}$ and an ellipticity of 0.44 . SC3 observes righthand waves with $\theta_{k B}$ between 47 and $56^{\circ}$ and ellipticities between 0.38 and 0.60 until 05:45:47, but after this SC3 observes left-hand waves until 05:45:51. The ellipticities for these left-hand waves vary between 0.37 and 0.67 and $\theta_{k B}$ between 28 and $45^{\circ}$. 
Cluster_data:17.3.2001 - GSM


$$
\begin{array}{r}
-1.8 \\
0.4
\end{array}
$$

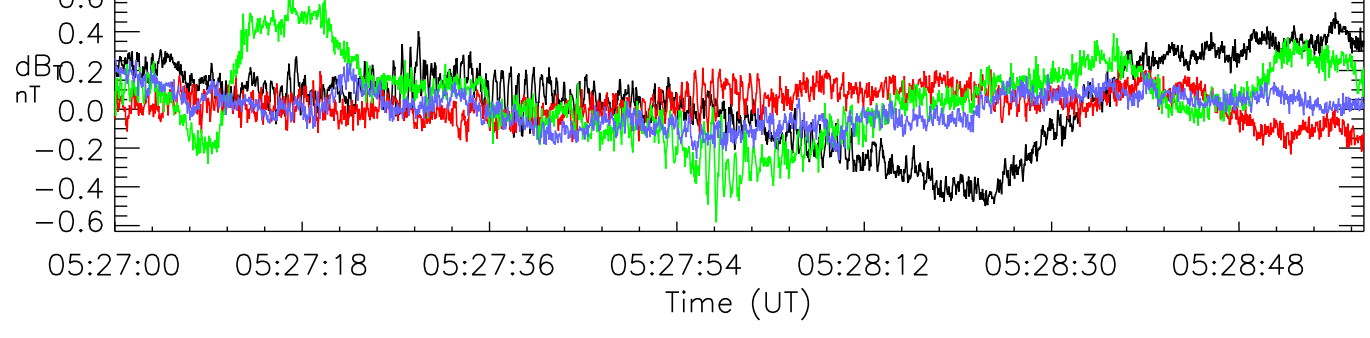

Fig. 9. De-trended magnetic field components between 05:25-05:27 (a) and between 05:27-05:29 (b). 

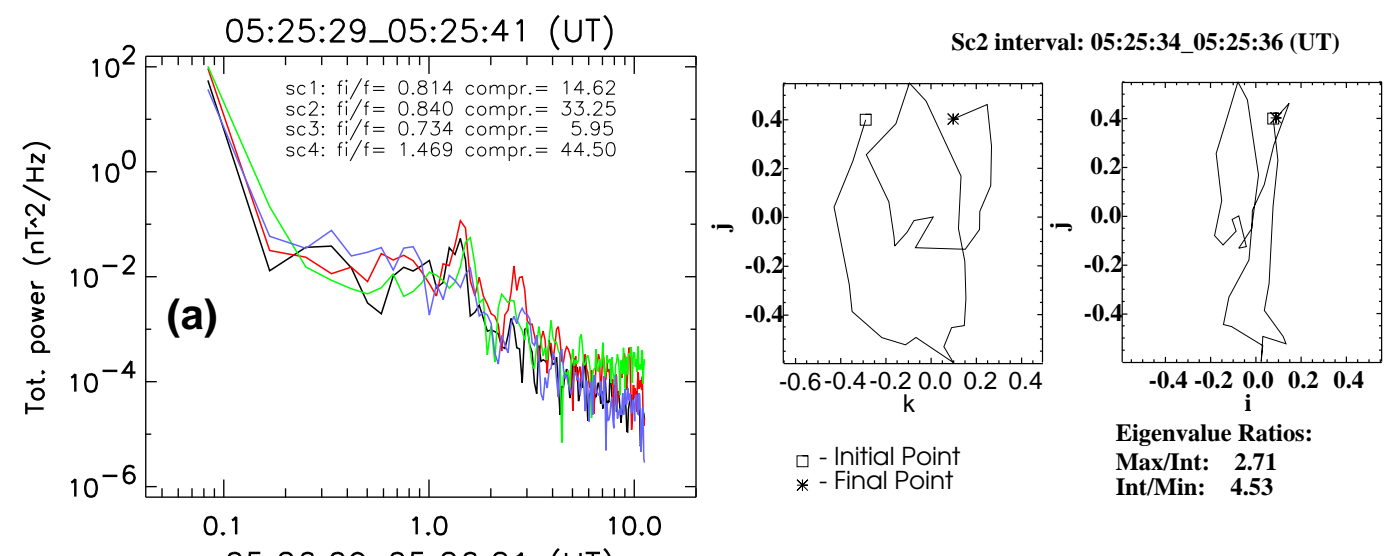

Eigenvectors:

$$
\begin{aligned}
& \text { j = [-0.59; } \\
& \text { 0.69; }
\end{aligned}
$$

Eigenvalue Ratios:

Max/Int: $\quad 2.71$

Int/Min: 4.53

Angles with B:

iB: 136.5

jB: 83.0

kB: 47.4
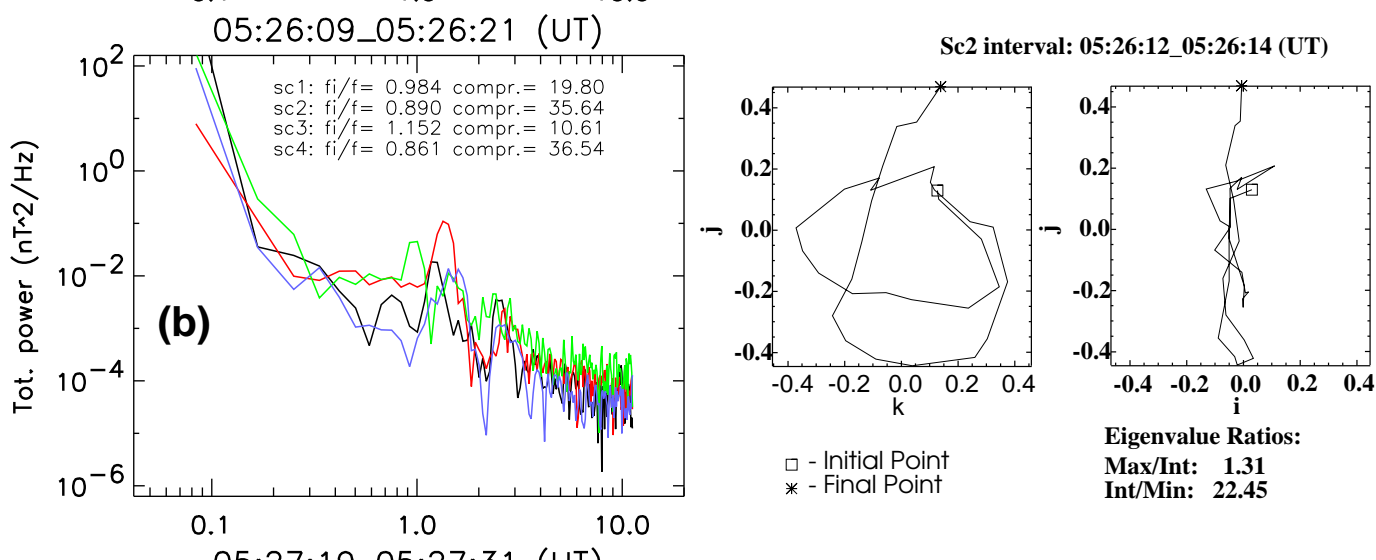

Eigenvectors:

$\mathbf{j}=[\mathbf{0 . 8 0}$;

0.06;

$-0.60]$

$\mathbf{k}=[-\mathbf{0 . 0 2}$;

$1.00 ;$

$=[0.60$;

-0.05;

\section{$\square$ - Initial Point \\ * - Final Point}

Eigenvalue Ratios:

Max/Int: 1.31

Int/Min: 22.45

Sc1 interval: 05:27:30_05:27:31 (UT)


Eigenvectors:

$\mathbf{j}=[0.86$;

$-0.14 ;$
$-0.48]$

$\mathrm{k}=[\mathbf{0 . 1 7}$

0.99 ;

0.02]

$i=[0.48$;

$-\mathbf{- 0 . 0 9}$

0.87]

Angles with B:

iB: 169.6

jB: 92.1

kB: $\mathbf{7 9 . 8}$
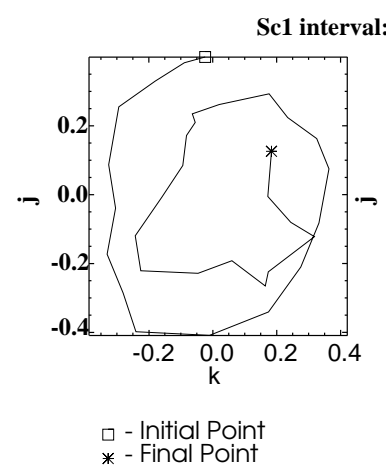

Eigenvectors:

j = [-0.27;

0.89;

$\mathrm{k}=[-\mathbf{0 . 8 7}$;

-0.39 ;

0.30]

$\mathrm{i}=[0.41$

$\mathbf{- 0 . 2 4}$

Angles with B:

iB: 175.5

jB: 86.7

kB: 87.0
Int/Min: $\mathbf{5 0 . 2 5}$
Eigenvalue Ratios: 10.0

Fig. 10. Total power of magnetic field fluctuations (left) at 05:25:29-05:25:41 (a), at 05:26:09-05:26:21 (b), at 05:27:19-05:27:31 (c) and at 05:27:54-05:28:06 (d). Hodograms of the wave magnetic field (right) observed by SC2 beteeen 05:25:34-05:25:36 (a), betweeen 05:26:12-05:26:14 (b), and by SC1 between 05:27:30-05:27:31 (c) and at 05:27:56-05:27:57 (d). 
In the second interval SC1, SC2, and SC3 observe peaks in power at $R=0.8,1$ and 0.8 , respectively. $\mathrm{SC} 4$ sees nothing in the way of wave power. While the waves are transverse, for $\mathrm{SC} 1$ we find $C=3$, the lowest ratio during this cusp crossing. Hodograms from SC1 between 05:46:27 and 05:46:28 UT show a right-hand polarized wave with $\theta_{k B}=11^{\circ}$ and ellipticity of 0.9 . SC3 does not observe any clearly polarized waves with ellipticities greater than 0.5. SC1 and SC2 observe five right-handed wave intervals with ellipticities between $0.55-$ 0.91 and $\theta_{k B}$, between 11 and $55^{\circ}$. SC1 also observes a lefthanded wave with ellipticity of 0.75 and $\theta_{k B}=39^{\circ}$. The first harmonic is also clearly observable by all 3 spacecraft.

In the third interval SC 2 and SC4 observe peaks at $R=0.82$ and 0.78 , respectively. The waves are transverse and the first harmonic is observed by both spacecraft. Surprisingly, SC4 again observes a left-handed wave between 05:47:19 and $05: 47: 21 \mathrm{UT}$ with $\theta_{k B}=10^{\circ}$ and with ellipticity of 0.71 . Between 05:47:18 and 05:47:30 UT SC2 sees right-handed waves, SC1 both left- and right-handed waves and SC3 a lefthand wave. The propagation angles vary between 6 and $71^{\circ}$ and ellipticities between 0.57 and 0.83 .

During the last interval, SC2 observes a very strong peak at $R=0.9$ and $C=5$, and in addition to this peak, SC2 also sees harmonics at $\sim 2.2 f_{i}$ and at $\sim 3.3 f_{i}$, respectively. The wave is clearly right-handed polarized with ellipticity of 0.76 and $\theta_{k B}=34^{\circ}$. Between 05:49:13 and 05:49:19 UT SC2 observes 7 right-handed waves with ellipticities varying between 0.22 to 0.80 and $\theta_{k B}$ between 34 to $65^{\circ}$.

Figure 14 shows wave properties of 75 wave intervals observed by all 4 spacecraft during 05:45:00 and 05:49:30 UT in the same format as Fig. 11. We have included only intervals with ellipticities greater than 0.4. Compared to the previous wave interval at 05:25-05:29 UT, these waves propagate with a more oblique angle with respect to the background magnetic field. Again, we do not see any clear correlation between ellipticity and wave propagation angle.

\section{Discussion and conclusions}

We have presented a comprehensive study of wave properties as measured in the magnetic field in the high-altitude cusp on 17 March 2001, with emphasis on waves around the ion cyclotron frequency and their association with plasma flows and velocity shear. The observed waves are very incoherent and mostly transverse, and propagate over a wide range of angles with respect to the magnetic field. Both left- and righthand polarised waves are detected. There was little or no correlation between the waves seen at spacecraft pairs.

In the interval of strongest plasma flow, the waves had amplitudes between 2 and $5 \mathrm{nT}$ and their occurrence correlated with enhanced plasma flows. We also noted the presence of velocity shear on the scale of the spacecraft separation, with spacecraft located in regions of rapid flow observing waves with frequencies close to or just below the local ion cyclotron frequency in the spacecraft frame. While they should

\section{Wave properties between 5:25-5:29 UT}

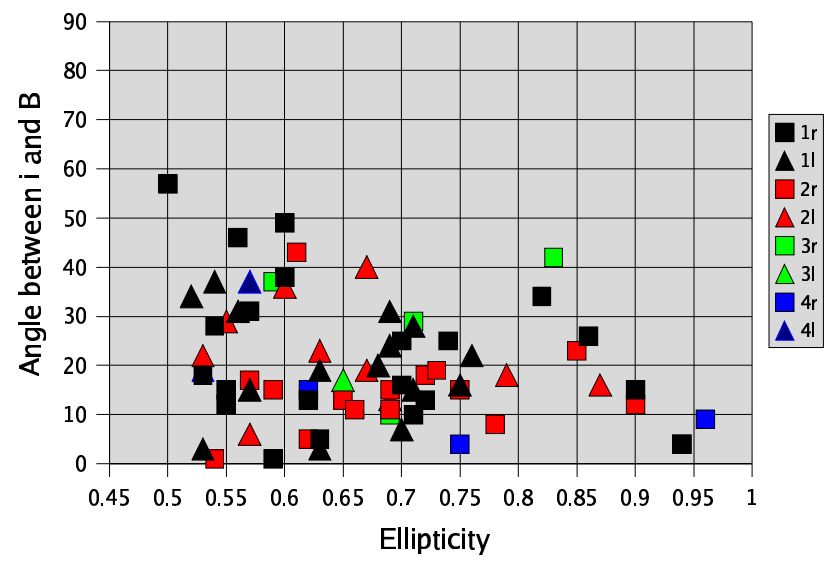

Fig. 11. The ellipticity of the waves between 05:25 and 05:29 UT as a function of the angle between the minimum variance direction and the ambient magnetic field. Squares and triangles represent righthand and left-hand polarized waves, respectively, as seen in 1-2-s intervals at each of the spacecraft. The usual colour coding is used.

be left-handed Alfvén/ion cyclotron modes, we also found right-hand waves.

In the stagnant cusp, the waves have lower amplitudes (below $1 \mathrm{nT}$ ), consistent with a lower level of free energy and are more coherent. The waves were predominately right-handed, with frequencies above the ion cyclotron frequency, but lefthanded waves were also seen frequently in this regime. If these are not the slow/sound modes, the observations of lefthand polarized waves above the ion cyclotron frequency are a puzzle, since Doppler effects cannot change the wave polarity. An interesting feature of this stagnant cusp was the detection of the first harmonic of the dominant frequency.

Two questions arise immediately from these results: how typical are they of the cusp in general, and what is the origin of the waves? The former requires an analysis of the full Cluster data base which has yet to be undertaken, but we note here that Nykyri et al. (2003) have analyzed two other cusp crossings from March 2002 with similar IMF conditions and also find magnetic field fluctuations close to ion cyclotron frequency, as well as a wide range of polarisations, ellipticities and propagation angles. It is also worth noting that in the absence of plasma data, the cusp can usually be identified by the onset of magnetic turbulence (Dunlop et al., 2000), again suggesting that it is quite ubiquitous. Also, a preliminary examination of the stagnant exterior cusp (SEC) of 4 February 2001 (Lavraud et al., 2002) reveals significant magnetic field turbulence there, although the plasma beta is then well in excess of unity. Finally, we note that a similarly complex picture emerged from the recent work of Le et al. (2001), who used data from the Polar spacecraft, albeit in different parts of the cusp due to the differing orbits.

The origin of the waves in this case appears to be related to the field-aligned sheared plasma flows generated by lobe reconnection, although a different origin might be expected 


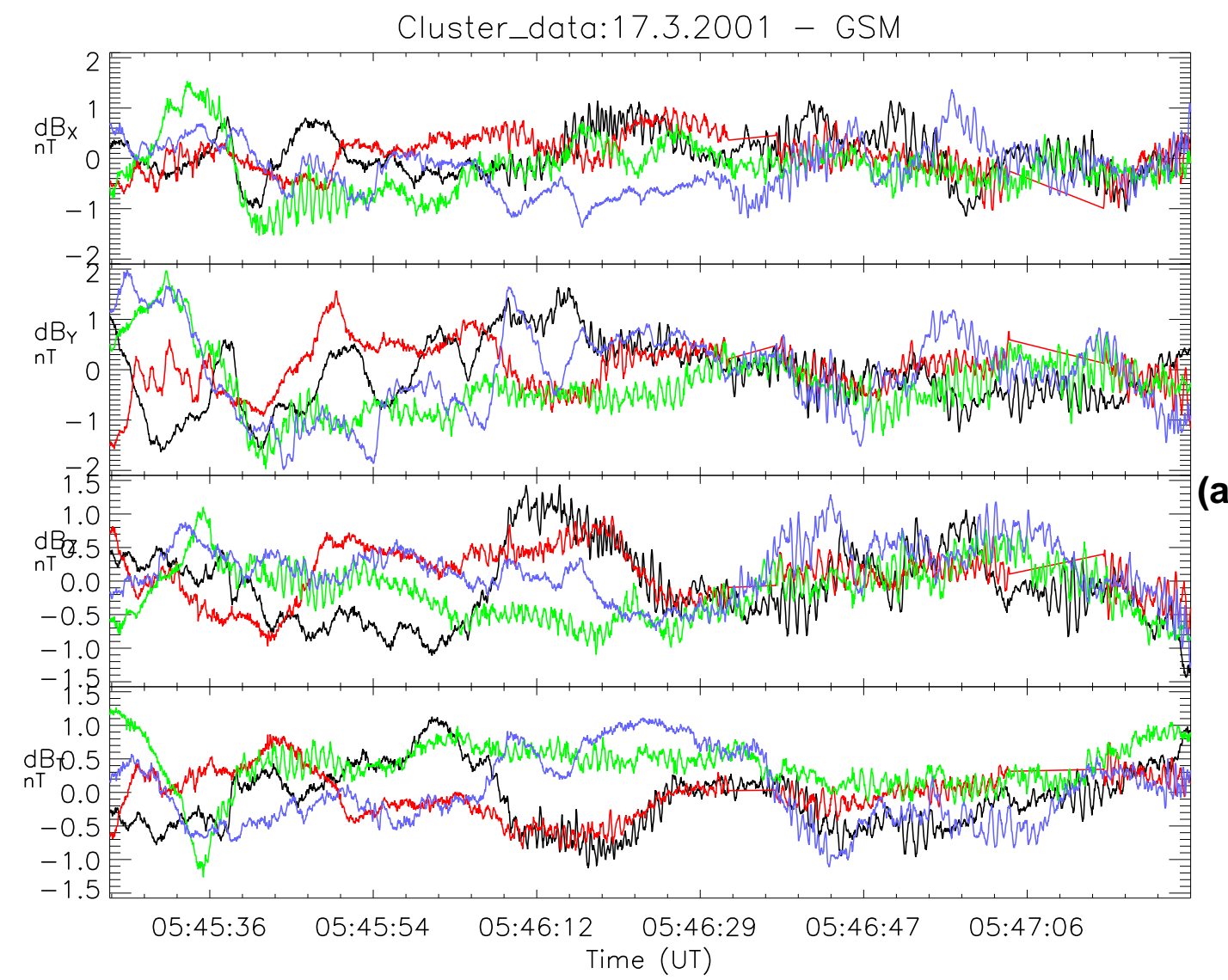

(a)

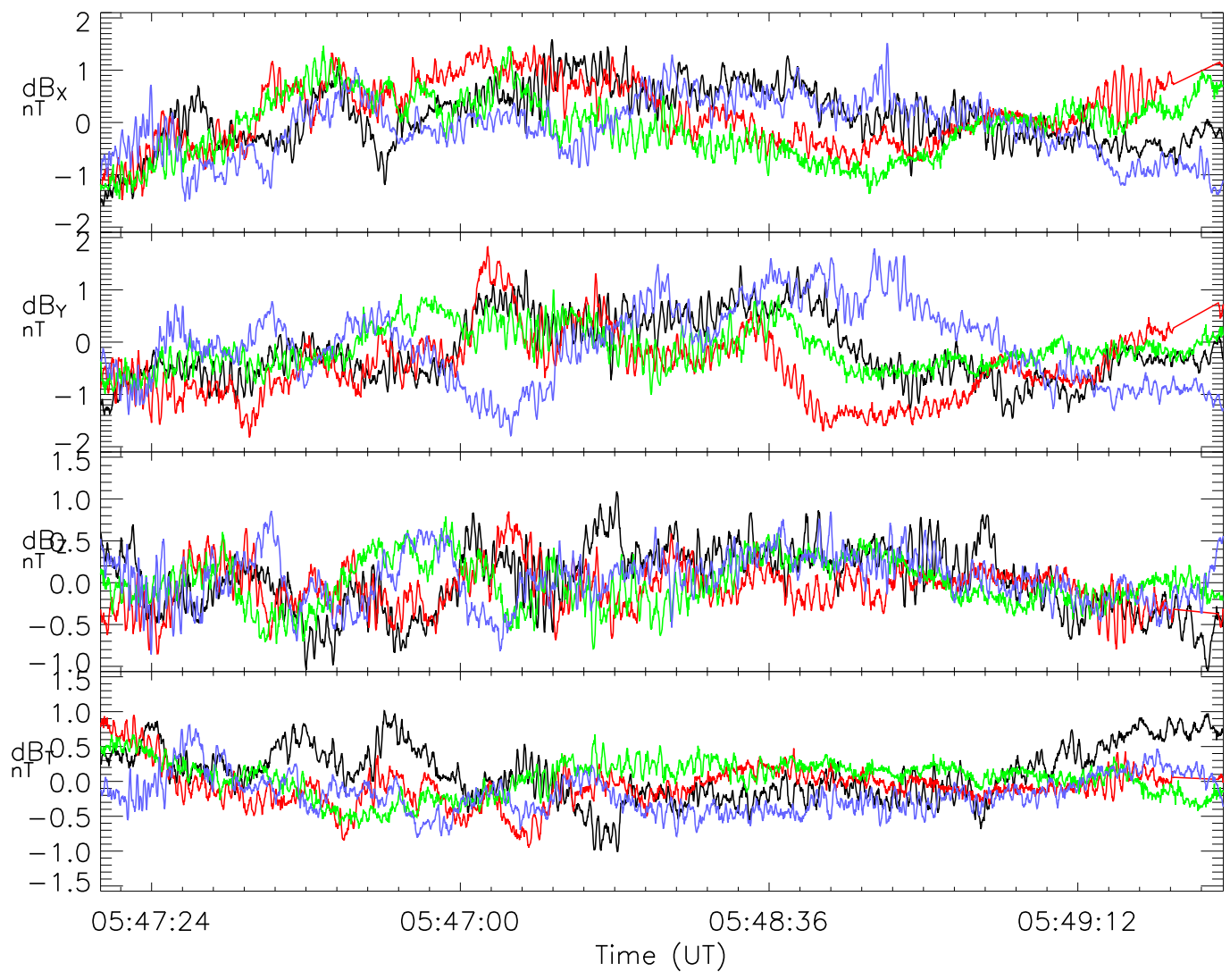

(b)

Fig. 12. De-trended magnetic field oscillations between 05:45-05:47 (a) and 05:47-05:49 (b). 

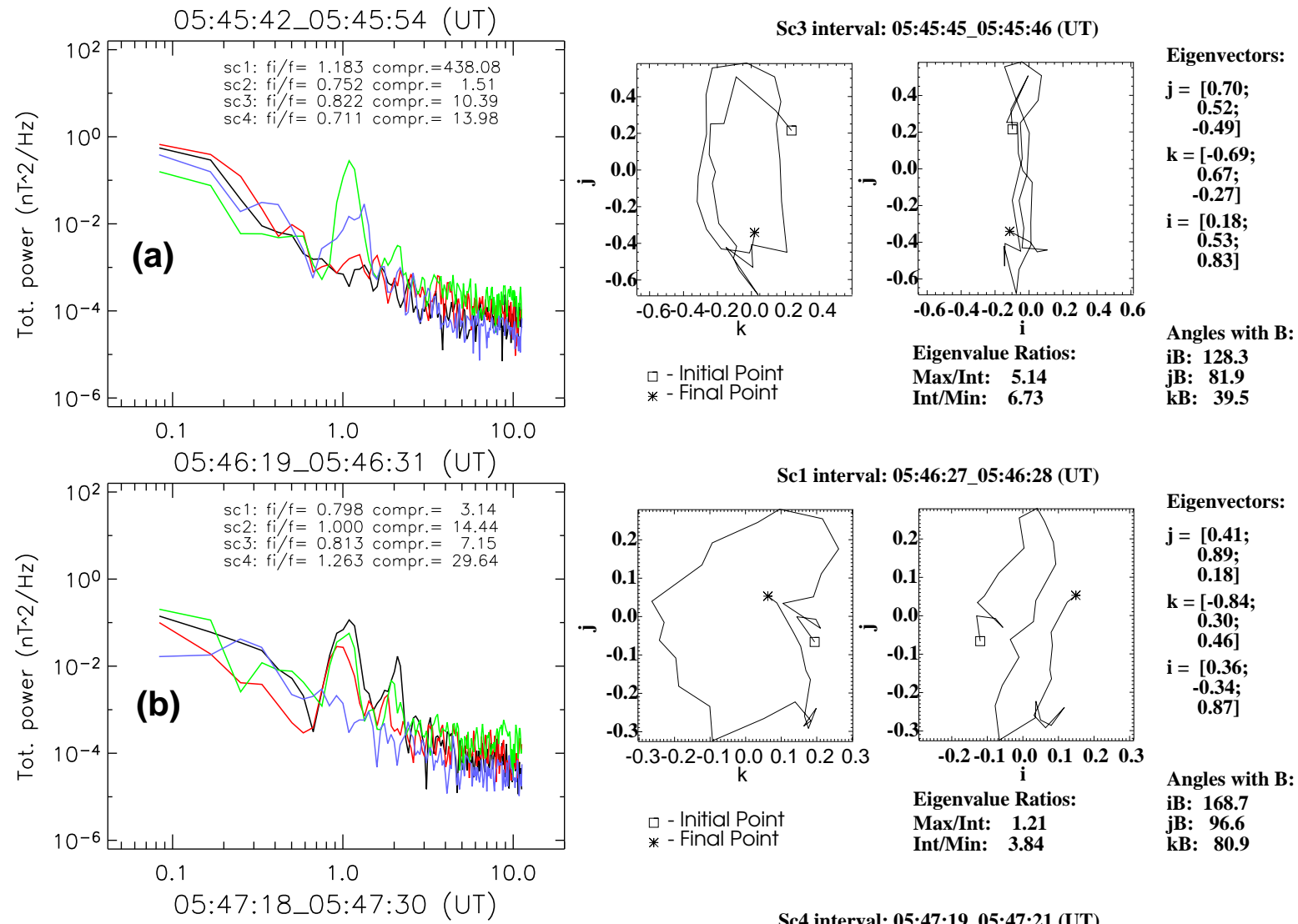

Eigenvectors:

$\mathbf{j}=[\mathbf{0 . 4 1}$;

$$
\text { 0.89; }
$$

$$
\text { 0.18] }
$$

$\mathrm{k}=[-\mathbf{0 . 8 4}$ 0.30;

$\mathrm{i}=[\mathbf{0 . 3 6}$;

-0.34;

0.87]

Angles with B:

\section{Eigenvalue Ratios: \\ iB: 168.7}

Max/Int: $1.21 \quad$ jB: 96.6

Int/Min: 3.84

kB: 80.9
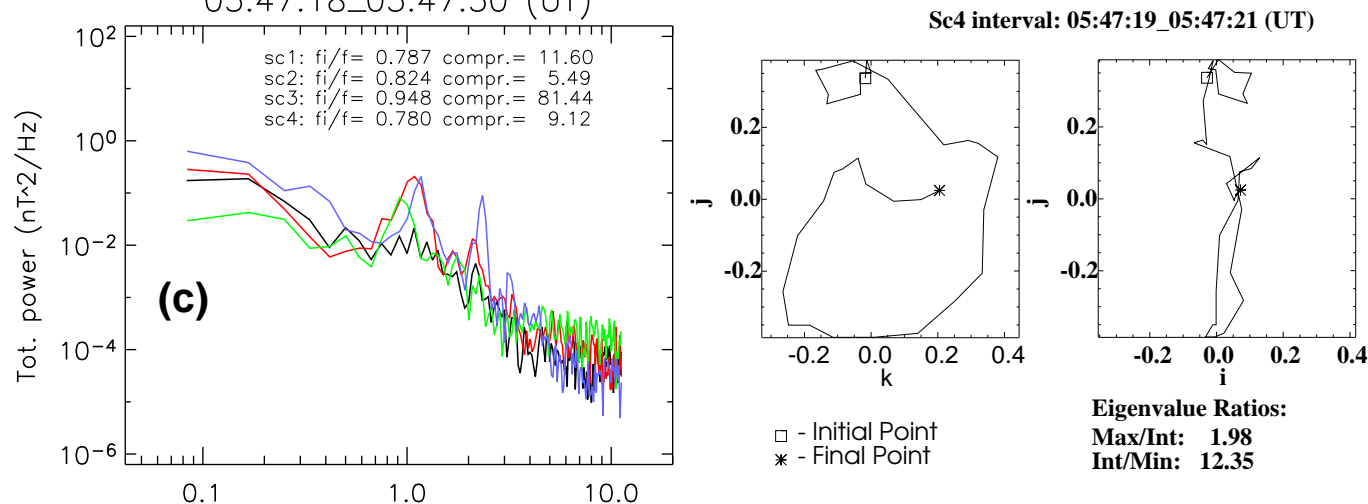

Eigenvectors:

$\mathbf{j}=[-0.46$;

$$
\text { 0.81; }
$$

$\mathrm{k}=[\mathbf{- 0 . 7 3}$

$\mathbf{- 0 . 5 7}$;

0.38]

$\mathrm{i}=[\mathbf{0 . 5 1}$;

$\mathbf{- 0 . 0 9 ;}$

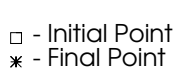

Eigenvalue Ratios:

Max/Int: 1.98

Angles with B:

iB: 169.5

jB: 80.5

kB: 94.6
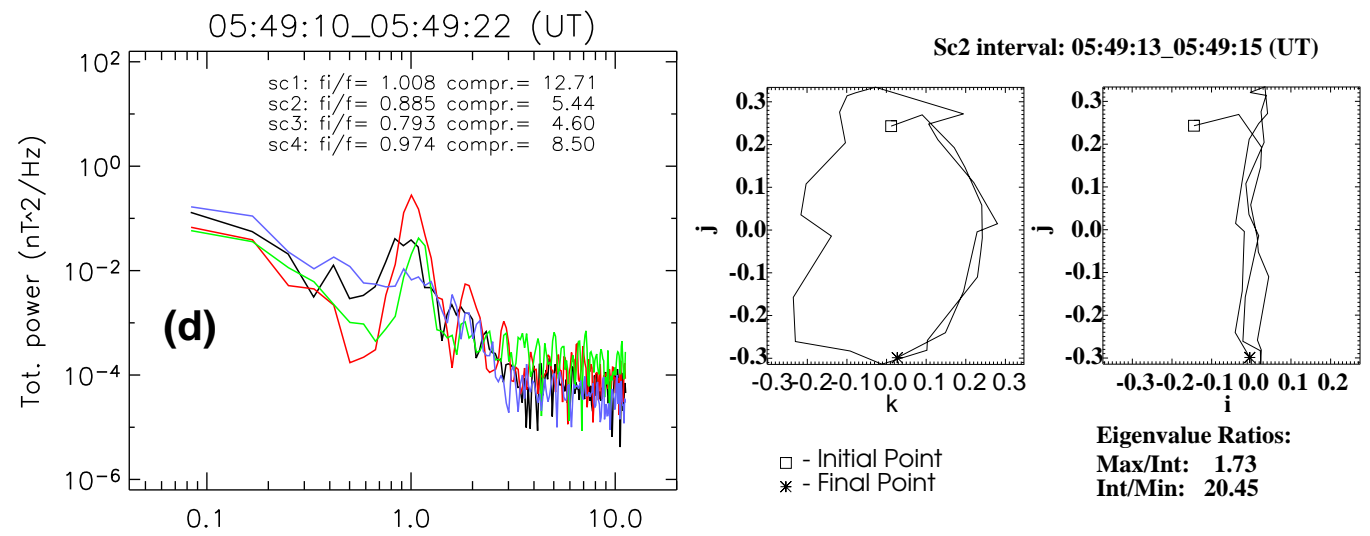

Eigenvectors:

$\mathbf{j}=[-\mathbf{0 . 5 4}$;

0.27;

$k=[0.76$;

0.57;

0.32]

$\mathrm{i}=[-\mathbf{- 0 . 3 7}$;

$0.78 ;$
$-\mathbf{- 0 . 5 1}]$

Angles with B:

iB: 33.6

jB: 104.7

kB: 119.5

Fig. 13. Total power of magnetic field fluctuations at 05:45:42-05:45:54 UT (a), 05:46:19-05:46:31 UT (b), 05:47:18-05:47:30 UT (c) and at 05:49:10-05:49:22 UT (d). On right-hand side are hodograms of the wave magnetic field observed by SC3 05:45:45-05:45:46 UT (a), by SC1 between 05:46:27-05:46:28 UT (b), by SC4 between 05:47:19-05:47:21 UT (c), and by SC2 between 05:49:13-05:49:15 UT (d). 


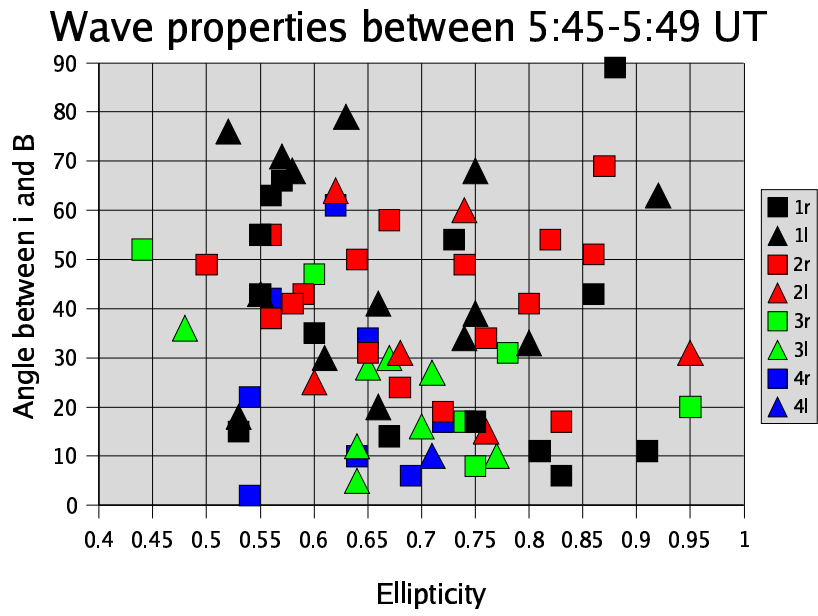

Fig. 14. Wave properties between 05:45-05:49 UT. Squares and triangles represent right-hand and left-hand polarized waves, respectively. The format is the same as Fig. 11.

for the SEC. However, without a more detailed survey of the cusp, we cannot say at present how general this result is, or indeed whether the waves are generated in-situ by the local velocity shear, or near a lobe reconnection site, and convected to the inner cusp. Addressing this question requires an analysis of the local ion and electron distribution functions.

We have noted a close association of these waves to velocity shear, and have noted occasions where the velocity changes very rapidly over the spacecraft separation scale. The velocity shear presumably arises from the lobe reconnection process and is a well-known source of plasma instabilities at around the ion cyclotron frequency (e.g. Ganguli and Palmadesso, 1988 and Ganguli et al., 1994). Alternatively, localised beams of electrons and/or ions with or without shear constitute a free energy source, and these too are well known as being unstable. However, if this is indeed the relevant process, it implies the injection of particles on relatively small scales above the cusp.

Finally, the role that these waves play in heating, scattering and transport in the cusp is unclear at this time. Their localised nature suggests that they may be generated and then damped in very small regions as opposed to filling the cusp with a quasi-homogeneous level of turbulence. The energy level in the waves is small, indicating that one should not expect significant plasma heating. Indeed, for a plasma density of $10 \mathrm{~cm}^{-3}$, complete dissipation of waves with amplitude of $5 \mathrm{nT}$ would lead to a temperature increase of under $10^{5} \mathrm{~K}$ : inconsequential from the viewpoint of the measured temperatures in the cusp. Scattering is another matter: it is an energy-conserving process, so the waves may be significant from that viewpoint. However, in order to produce locally the ion energies in excess of $1 \mathrm{MeV}$ reported by Chen and Fritz (1998), one needs wavelengths of the order of $1.5 R_{E}$, well in excess of what is observed here, and of the order of the scale of the cusp.
Acknowledgements. Cluster work in the UK and France is supported by PPARC and CNES, respectively. PC also thanks PPARC for the award of a Senior Research Fellowship. We would also like to thank J. P. Eastwood for comments.

Topical Editor T. Pulkkinen thanks J. Blecki and R. L. Kessel for their help in evaluating this paper.

\section{References}

Balogh, A., Dunlop, M. W., Cowley, S. W. H., Southwood, D. J., Thomlinson, J. G., and magnetometer team, C.: The Cluster magnetic field investigation, Space Sci. Rev., 79, 65, 1997.

Balogh, A., Carr, C. M., Acuna, M. H., Dunlop, M. W., Beek, T. J., Brown, P., Fornangon, K.-H., Georgescu, E., Glassmeier, K.-H., Harris, J., Musmann, G., Oddy, T., and Schwingenschuh, K.: The Cluster magnetic field investigation: overview of in-flight perfomance and initial results, Ann. Geophys., 19, 1207, 2001.

Cargill, P. J., Dunlop, M. W., Lavraud, B., Elphic, R. C., Holland, D. L., Nykyri, K., et al.: CLUSTER encounters with the high altitude cusp: boundary structure and magnetic field depletions, Ann. Geophys., 22, 1739-1754, 2004.

Chen, J. S. and Fritz, T. A.: Correlation of cusp mev helium with turbulent ULF power spectra and its implications, 1998.

Dunlop, M. W., Cargill, P. J., Stubbs, T. J., and Woolliams, P.: The high altitude cusps: Heos 2, J. Geophys. Res., 105, 27 509, 2000.

Fuselier, S. A., Trattner, K. J., and Petrinec, S. M.: Cusp observations of high- and low-latitude reconnection for northward interplanetary magnetic field, J. Geophys. Res., 105, 253, 2000.

Ganguli, G. and Palmadesso, P. J.: Electrostatic ion instabilities in the presence of parallel currents and transverse electric fields, Geophys. Res. Lett., 15, 103, 1988.

Ganguli, G., Keskinen, M. J., Romero, H. et al.: Coupling of microprocesses and macroprocesses due to velocity shear: An application to the low-altitude ionosphere, J. Geophys. Res., 99, 8873, 1994.

Johnstone, A. D., Burge, S., Carter, P. J., Coates, A. J., Coker, A. J., Fazakerley, A. N., et al.: Peace: A plasma electron and current experiment, Space Sci. Rev., 79, 351, 1997.

Kessel, R. L., Chen, S.-H., Green, J. L., Fung, S. F., Boardsen, S. A., Tan, L., Eastman, T. E., Craven, J. D., and Frank, L. A.: Evidence of high-latitude reconnection during northward IMF: Hawkeye observations, Geophys. Res. Lett., 23, 583, 1996.

Krauss-Varban, D., Omidi, N., and Quest, K. B.: Mode properties of low-frequency waves: Kinetic theory versus Hall-MHD, J. Geophys. Res., 99, 5987, 1994.

Lavraud, B., Dunlop, M. W., Phan, T. D., et al.: Cluster observations of the exterior cusp and its surrounding boundaries under northward IMF, Geophys. Res. Lett., 29, (20), 2002.

Lavraud, B., Phan, T. D., Dunlop, M. W., Taylor, M. G. G. T., Cargill, P. J., Bosqued, M. J., Dandouras, I., Réme, H., Sauvaud, J. A., Balogh, A., and Fazakerley, A.: The exterior cusp and its boundary with the magnetosheath under northward IMF: Cluster multi-event analysis, Surveys in Geophysics, in press, 2004.

Le, G., Blanco-Cano, X., Russell, C. T., Zhou, X.-W., Mozer, F., Trattner, K. J., Fuselier, S. A., and Anderson, B. J.: Electromagnetic ion cyclotron waves in the high-altitude cusp: Polar observations, J. Geophys. Res., 106, $19067,2001$.

Nykyri, K., Cargill, P. J., Lucek, E., Horbury, T. S., Balogh, A., Lavraud, B., Dandouras, I., and Rème, H.: Ion cyclotron waves in the high altitude cusp: Cluster observations at varying spacecraft separations, Geophys. Res. Lett., 30, (24), 2263, 2003. 
Paschmann, G., Haerendel, G., Sckopke, N., Rosenbauer, H., and Hedgecock, P. C.: Magnetic field characteristics of the distant polar cusp near local noon: the entry layer, J. Geophys. Res., 81, 2883, 1976.

Rème, H., Aoustin, C., Bosqued, J. M., Dandouras, I., Lavraud, B., et al.: First multispacecraft ion measurements in and near the Earth's magnetosphere with the identical Cluster ion spectrometry (CIS) experiment, Ann. Geophys., 19, 1303, 2001.

Rezeau, L., Roux, A., and Russell, C. T.: Characterization of smallscale structures at the magnetopause from isee measurements, J. Geophys. Res., 98, 179, 1993.

Russell, C. T., Le, G., and Petrinec, S. M.: Cusp observations of high-and low-latitude reconnection for northward imf: An alternate view, J. Geophys. Res., 105, 5489, 2000.

Savin, S. P., Romanov, S. A., Fedorov, A. O., Zelenyi, L., Klimov, S. I., Yermolaev, Y. I., Budnik, E. Y., Nikolaeva, N. S., Russell, C. T., Zhou, X. W., Urguhart, A. L., and Reiff, P. H.: The cusp/magnetosheath interface on may 29, 1996: Interball-1 and Polar observations, Geophys. Res. Lett., p. 3015, 1998.

Savin, S. P., Zelenyi, L., Romanov, S. A., Sandahl, I., Pickett, J., Amata, E., Avanov, L., Blecki, J., et. al.: Magnetosheath-cusp interface, Ann. Geophys., 22, 183, 2004.

Scarf, F. L., Fredricks, R. W., Neugebauer, M., and Russell, C. T.: Plasma waves in the day side polar cusp, 1. magnetopause and and polar magnetosheath, J. Geophys. Res., 79, 511, 1972.
Scudder, J. D., Mozer, F. S., Maynard, N. C., and Russell, C. T.: Fingerprints of collisionless reconnection at the separator, J. Geophys. Res., 107, SMP13-1 to SMP13-38, 2002.

Sonnerup, B. U. Ö. and Scheible, M.: Minimum and maximum variance analysis, in Analysis methods for multi-spacecraft data, ISSI Scientific Report, edited by Paschmann, G. and Daly, P. W., p. 185, The International Space Science Institute, Hallerstrasse 6, CH-3012 Bern, Switzerland, 1998.

Trattner, K. J., Fuselier, S. A., Peterson, W. K., Sauvaud, J.-A., Stenuit, H., Dubouloz, N., and Kovrazhkin, R. A.: On spatial and temporal structures in the cusp, J. Geophys. Res., 104, 28411 , 1999.

Tsyganenko, N. A.: A magnetospheric magnetic field model with a warped tail current sheet, Planet. Space Sci., 37, 5, 1989.

Vontrat-Reberac, A., Bosqued, J. M., Taylor, M. G., Lavraud, B., Fontaine, D., Dunlop, M. W., Laakso, H., Cornilleau-Werhlin, N., Canu, P., and Fazarkerley, A.: Cluster observations of the high-altitude cusp for northward interplanetary magnetic field: a case study, J. Geophys. Res., 108, (A9), 2003.

Zhou, X. W., Russell, C. T., Le, G., Fuselier, S. A., and Scudder, J. D.: The polar cusp location and its dependence on dipole tilt, Geophys. Res. Lett., 26, 429, 1999.

Zhou, X. W., Russell, C. T., Le, G., Fuselier, S. A., and Scudder, J. D.: Solar wind control of the polar cusp at high altitude, J. Geophys. Res., 105, 245, 2000. 